\title{
22. GEOCHEMISTRY AND PETROGENESIS OF BASALTS FROM HOLES 767C, 770B, AND 770C, CELEBES SEA ${ }^{1}$
}

\author{
T. E. Smith, ${ }^{2}$ C. H. Huang, ${ }^{3}$ and F. G. Sajona ${ }^{4}$
}

\begin{abstract}
Major-, trace-, and rare-earth element analyses are presented from a suite of basaltic rocks from the basement of the Celebes Sea. The major elements and trace-elements were determined by X-ray fluorescence techniques, and the rare-earth elements were analyzed by instrumental neutron activation analysis. Compositionally the Celebes Sea basalts are very similar to typical normal mid-ocean ridge basalts, such as those described from the Indian Ocean triple junction. Petrogenetic modeling shows that all of the basalts analyzed can be formed by $10 \%$ to $20 \%$ partial melting of a light rare-earth element-depleted spinel lherzolite followed by fractional crystallization of mixtures of olivine, plagioclase, and iron oxide. The Celebes Sea is interpreted as a fragment of the basement of the Jurassic Argo abyssal plain trapped during the Eocene to the north of Australia.
\end{abstract}

\section{INTRODUCTION}

The origin of the Celebes Sea (Fig. 1), a small ocean basin, is in dispute. Lee and McCabe (1986) have suggested that it represents a trapped part of a once larger ocean basin, but it is also possible that the basin formed by back-arc spreading. There are commonly significant differences in the major- and trace-element compositions of volcanic rock suites erupted during the formation of a major ocean as compared to those formed in back-arc environments. Among the objectives of ODP Leg 124 was to use the nature and composition of the basement of the Celebes Sea, together with the data on the stratigraphy and sedimentology of the overlying sediments, to help determine its origin.

The major- and trace-element data published for the volcanic rocks making up the basement of the Celebes Sea (Shipboard Scientific Party, 1990), supplemented by additional trace- and rare-earth element (REE) data, are used in this study. The data, together with a summary of the mineralogy and petrography of basement samples recovered during Leg 124, are compared with the characteristics of well described suites which have erupted in known tectonic environments. The geotectonic significance, in the evolution of the southeast Pacific, of the origin of the Celebes Sea implied by this comparison, is evaluated.

\section{PETROGRAPHY AND GEOCHEMISTRY}

\section{Recovery}

Three holes, 767C, 770B, and 770C, were drilled to basement in the Celebes Sea, on ODP Leg 124. Only $42 \mathrm{~cm}$ of core, consisting of four pieces, were recovered from the basement of Hole $767 \mathrm{C}$. About $53.18 \mathrm{~m}$ of igneous rocks were penetrated at the bottom of Hole 770B and core recovery averaged

\footnotetext{
${ }^{1}$ Silver, E. A., Rangin, C., von Breymann, M. T., et al., 1991. Proc. ODP, Sci. Results, 124: College Station, TX (Ocean Drilling Program).

${ }^{2}$ Department of Geology, University of Windsor, Windsor, Ontario, N9B 3P4, Canada.

${ }^{3}$ Department of Geology, Laurentian University, Sudbury, Ontario, P3E 2C6, Canada.

${ }_{4}^{4}$ Petrolab, Mines and Geosciences Bureau, North Avenue, Diliman, Quezon City, Philippines.
}

$47.0 \% ; 106.30 \mathrm{~m}$ were penetrated at the bottom of Hole $770 \mathrm{C}$ with an average recovery of $42.8 \%$.

The original subdivision of the basement rocks recovered from Holes 770B and 770C, was made using their lithological and structural characteristics (Shipboard Scientific Party, 1990, Fig. 14, pp. 359-369). Minor revisions have been made to the grouping of the rocks into units using more detailed mineralogical, petrographic, and geochemical characteristics, and geochemical logs (Shipboard Scientific Party, 1990, pp. 379-381). In the subdivision of the rocks recovered from Hole $770 \mathrm{~B}$, the original Units 2 and 3 are amalgamated into a new Unit 2, and the original Units 4, 5, and 6 are combined to make up the new Unit 3 . In Hole $770 \mathrm{C}$ the only change made to the original subdivision involves the incorporation of original Units 3 and 4 into the new Unit 3 . The original Units 5, 6, 7, 8 , and 9 are renumbered as new Units $4,5,6,7$, and 8 . The thicknesses and positions within the cores of the new units are described in Table 1.

\section{Sample Description}

The mode of occurrence of the samples from the basement of Hole $767 \mathrm{C}$ is indeterminate. The rocks consist of hypocrystalline, cryptocrystalline to fine-grained basalts with variolitic, spinifex, fasciculate, and intersertal textures indicative of the rapid quenching of a superheated basaltic magma (Augustithius, 1979, pp. 65-67, and Walker et al., 1979, Fig. 5). Their groundmass comprises intergrown skeletal, lath, and prismatic crystals of olivine, plagioclase, clinopyroxene, and grains of $\mathrm{Fe}$-Ti oxide in variable proportions, set in dense unresolvable mineral aggregates and devitrified glass. The olivines are commonly pseudomorphed by green and red clays whereas plagioclase and clinopyroxene appear to be unaltered. Vesicles are ubiquitous and generally filled with clays. Fractures filled with clay, carbonate, iron ore and chalcedony also occur.

The basement rocks recovered from Holes $770 \mathrm{~B}$ and $770 \mathrm{C}$ occur as pillow lavas and pillow breccias, as massive, commonly brecciated lavas, as sills, and as very small dikes. All of the samples studied are basalts and their mineralogical and petrographic characteristics are summarized in Table 1. The majority of these rocks comprise plagioclase $\left(\mathrm{An}_{60-70}\right.$, estimated optically) and olivine phenocrysts set in a matrices of plagioclase $\left(\mathrm{An}_{50-70}\right)$, clinopyroxene, glass and/or devitrified mesostasis. 


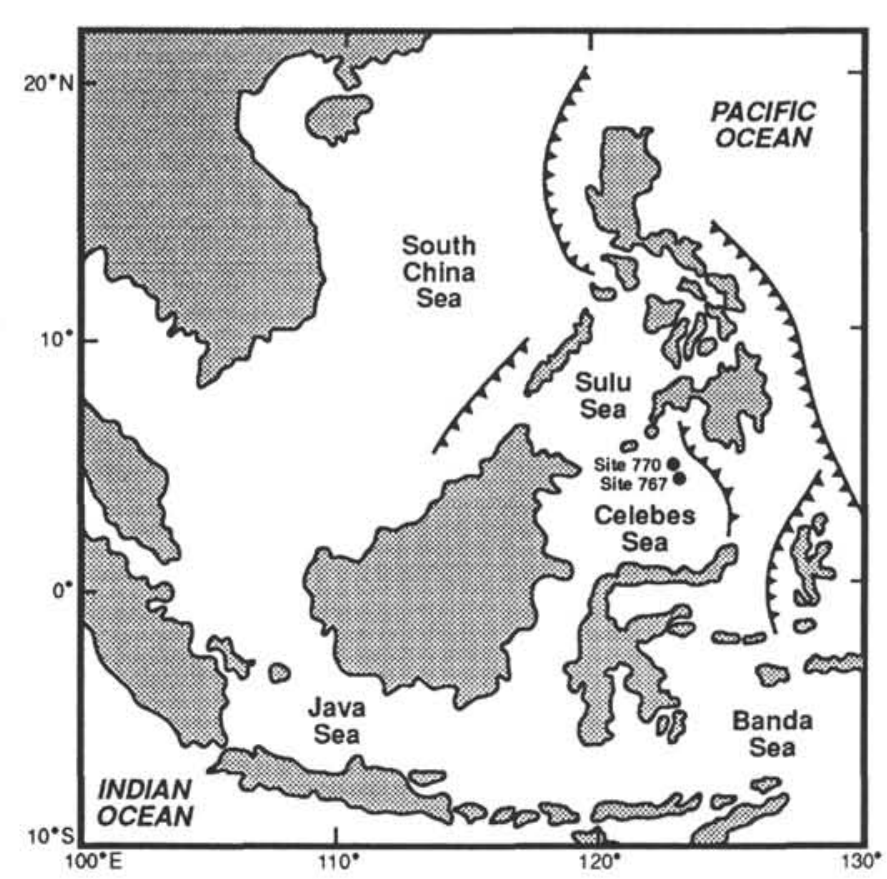

Figure 1. Simplified tectonic map showing the locations of Sites 767 and 770 , Celebes Sea.

The phenocryst assemblages and petrographic characteristics of these rocks suggest that in the majority of cases crystallization started with olivine and plagioclase as the liquidus and near-liquidus phases. In Unit 6 the aphyric to plagioclase phyric dolerite (Table 1), plagioclase is on the liquidus. Following the crystallization of olivine and plagioclase phenocrysts, the remainder of the melt was quenched to yield a mixture of skeletal crystals of plagioclase, clinopyroxene, and iron oxide set in a glassy mesostasis, in all samples. The textures, mineralogy, and crystallization history of these olivine-plagioclase phyric basalts are typical of, but not exclusive to, mid-ocean ridge basalts (MORB's) (see Basaltic Volcanism Study Project 1981, pp. 134-138).

\section{METHODS}

Details of the shipboard analytic methods are given in Shipboard Scientific Party (1990, pp. 28-29). The additional trace-element analyses were carried out at Laurentian University using a Philips PW1400 X-ray Spectrometer. The rareearth element contents were determined by instrumental neutron activation analysis (INAA) at X-Ray Assay Laboratories, Toronto. Full details of the methods are given in Abbott and Smith (1978) and Huang and Smith (1983) for XRF, and Peck and Smith (1989) for XRF and INAA.

The precisions of all of the additional trace-element determinations, calculated using the average standard deviation for each element for several replicate analyses, are $5 \%$. The precisions of the REE determinations, calculated in the same manner, are 5\% for $\mathrm{La}, \mathrm{Nd}, \mathrm{Sm}, \mathrm{Eu}, \mathrm{Tb}, \mathrm{Yb}$, and $\mathrm{Lu}$, and $10 \%$ for $\mathrm{Ce}$.

Comparison of the additional trace-element analyses with those determined on board ship shows excellent agreement for samples from the same units that have similar Mg-numbers, with two exceptions (Table 3). There are considerable differences in the $\mathrm{Rb}$ concentrations of similar rocks that may be the results of alteration and/or analytical error. Therefore, $\mathrm{Rb}$ is not used in the discussion below. The values of $\mathrm{Nb}$ in similar rocks agree well at concentration levels above $5 \mathrm{ppm}$, but at lower concentrations the shipboard values, are about half of those determined at Laurentian University on similar samples (Table 3). $\mathrm{Nb}$ is immobile during the alteration of the rocks and these differences are the result of analytical error. The Laurentian values are more precise than those determined on board ship, which show large errors (Shipboard Scientific Party, 1990, p. 265), and are therefore used in evaluating the petrogenesis of the basalts.

\section{ALTERATION}

All of the samples studied have undergone mineralogical alteration (Shipboard Scientific Party, 1990, Hole 767C Table 17, Holes $770 \mathrm{~B}$ and $770 \mathrm{C}$ Tables 10 and 11). The effects of this alteration on the chemical composition of the rocks are evaluated below. See Table 2 . Sub-alkaline basalts generally contain $<1.0 \%$ water and generally do not contain either normative nepheline $(\mathrm{Ne})$ or corundum $(\mathrm{C})$. Thus, basalt samples with $>2.0 \%$ loss on ignition (LOI) and those containing normative $\mathrm{C}$ or Ne should not be used in classification or petrogenetic studies without justification. None of the samples recovered contains normative $C$ and samples 2.5 and 3.1 (for sample numbers see Table 3$)$ contain normative $\mathrm{Ne}(0.49 \%$ and $1.26 \%$, respectively) (Shipboard Scientific Party, 1990, Holes 770B and 770C Table 15). Samples 3.1, 3.2, 3.5, 5.1, and 6.1 contain $>2.0 \%$ LOI (Shipboard Scientific Party, 1990, Holes 770B and 770C Table 13). They are included in the study because their multielement patterns are essentially identical with samples containing $<2 \%$ LOI (see below). In addition, these basalts may have formed in a back-arc basin environment and their higher than average volatile contents could be original (Saunders and Tarney, 1984; Rauterschlien et al., 1985).

Plots (Beswick and Sourcie, 1978) of the major-element chemistry, determined on board ship, of the basement rocks of the Celebes Sea suggest that there have been some changes in the composition of the volcanic sequence during alteration. The changes include slight increases in the original $\mathrm{K}_{2} \mathrm{O}$ contents, and small decreases in the original $\mathrm{Na}_{2} \mathrm{O}$ contents. The diagrams also suggest that the $\mathrm{SiO}_{2}, \mathrm{MgO}, \mathrm{CaO}$, and $\mathrm{Fe}_{2} \mathrm{O}_{3}{ }^{\mathrm{t}}$ (total iron expressed as $\mathrm{Fe}_{2} \mathrm{O}_{3}$ ) contents were relatively unaffected by alteration and are close to the original values (Fig. 2 contains two representative plots selected to illustrate the chemical changes that have occurred, See legend for further explanation).

The contents of $\mathrm{Rb}, \mathrm{Ba}, \mathrm{Th}, \mathrm{U}$, and $\mathrm{Sr}$ do not show systematic covariation with $\mathrm{Zr}$ or regular distribution on multielement plots. This suggests that these elements were mobile during alteration, and they are not used in the discussion below. The systematic covariation of the $\mathrm{Nb}, \mathrm{Y}, \mathrm{P}_{2} \mathrm{O}_{5}$, $\mathrm{TiO}_{2}, \mathrm{Ni}, \mathrm{Cr}, \mathrm{Sc}$, and the REE vs. $\mathrm{Zr}$, as demonstrated by the consistent elemental ratios and REE and multielement distribution (Tables 3 and 4, Figs. 3 and 4), suggests that these elements have been relatively immobile during alteration (see also Condie 1977; Gelinas et al., 1982; Ludden et al., 1982; Kokelaar et al., 1984; Pearce et al., 1984; Saunders and Tarney, 1984; Condie et al., 1985). Their original concentrations are little changed, even where major elements have been altered. The immobile element compositions of the rocks showing the most mineralogical alteration lie in the middle of the range of the suite and have very similar trace-element distributions to less altered samples. This indicates that the small amounts of volatiles that may have been added to the compositions of these rocks have diluted, or increased, the concentrations of the immobile minor- and trace-elements by limited amounts.

\section{CHEMISTRY}

The contents of $\mathrm{SiO}_{2}, \mathrm{Fe}_{2} \mathrm{O}_{3}{ }^{\mathrm{t}}$, and $\mathrm{MgO}$ are used, together with the immobile minor- and trace-element contents and 
Table 1. Summary of the mineralogy, petrography, and thicknesses of the lithological units identified in the basement of Holes 770B, 770C, Celebes sea.

Unit 1 Estimated thickness $18.42 \mathrm{~m}$ in Hole $770 \mathrm{~B}$ (base at $18 \mathrm{R}-3,74 \mathrm{~cm}$ ), $19.54 \mathrm{~m}$ in $\mathrm{Hole} 770 \mathrm{C}$ (base at $4 \mathrm{R}-1$, $14 \mathrm{~cm}$ ). Moderately Plagioclase-0livine Phyric Pillow Basalt Lavas and Breccias.
Hypocrystalline, fine to medium grained, hyalopilitic, intersertal to intergranular. PHENOCRYSTS

\begin{tabular}{|c|c|c|c|c|c|c|c|c|c|}
\hline olivine & $\begin{array}{l}\text { Size mm } \\
<1.0\end{array}$ & Composition & $\begin{array}{c}\text { Percentage } \\
1-10\end{array}$ & Composition & Percentage & $\begin{array}{l}\text { Size mm } \\
0.5-2.0\end{array}$ & $\begin{array}{l}\text { Shape } \\
\text { Spherical }\end{array}$ & $\begin{array}{c}\text { Filling } \\
\text { Clay, Limonite }\end{array}$ & $\begin{array}{c}\text { Percentage } \\
<2,0\end{array}$ \\
\hline $\begin{array}{l}\text { Plagioclase } \\
\text { Clinopyroxene }\end{array}$ & $<4.0$ & $A n_{50-70}$ & $2-15$ & $\mathrm{An}_{50 \cdot 70}$ & $\begin{array}{l}15-60 \\
0-55\end{array}$ & & $\begin{array}{l}\text { Lobate } \\
\text { Irregular }\end{array}$ & Calcite, silica & \\
\hline
\end{tabular}

UNIT 2 Estimated thickness $18.71 \mathrm{~m}$ in Hole $770 \mathrm{~B}$ (base at $20 \mathrm{R}-3,35 \mathrm{~cm}$ ), $9.46 \mathrm{~m}$ in Hole $770 \mathrm{C}$ (base at $5 \mathrm{R}-2$, top). Highly Plagioclase-0livine Phyric Pillow Basalt Lavas and Breccias. Hypocrystalline, cryptocrystalline to fine-grained, intersertal, intergranular, and sub-ophitic.

\begin{tabular}{|c|c|c|c|c|c|c|c|c|c|}
\hline PHENOCRYSTS & & & & GROUNDMASS & & VESICLES / & GDALES & & \\
\hline & Size $\mathrm{mm}$ & Composition & Percentage & Composition & Percentage & Size mm & Shape & Filling & Percentage \\
\hline Olivine & $<3.0$ & & $3-10$ & & & $<1.0$ & Spherical & Clay, Calcite & $<2.0$ \\
\hline $\begin{array}{l}\text { Plagioclase } \\
\text { clinopyroxene }\end{array}$ & $<5.0$ & $\mathrm{An}_{50-70}$ & $10-20$ & $\mathrm{An}_{60}$ & $\begin{array}{l}10-40 \\
0-30\end{array}$ & & & & \\
\hline Mesostasis & & & & & $0-70$ & & & & \\
\hline
\end{tabular}

UNIT 3 Estimated thickness $19.92 \mathrm{~m}$ in Hole $770 \mathrm{C}$ (base at $7 \mathrm{R}-1,82 \mathrm{~cm}$ ), $>16.05 \mathrm{~m}$ in Hole $770 \mathrm{~B}$ (base not reached). Moderate to Highly Plagioclase-0livine Phyric Amygdaloidal, Brecciated Lavas. Hypocrystalline to holocrystalline, fine- to medium-grained, intersertal, intergranular, intrafasciculate and sub-ophitic. PHENOCRYSTS

\begin{tabular}{|c|c|c|c|c|c|c|c|c|c|}
\hline 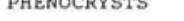 & & & & 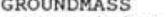 & & 2010 & & & \\
\hline Olivine & $\begin{array}{l}\text { Size man } \\
0.5-1.5\end{array}$ & Composition & $\begin{array}{l}\text { Percentage } \\
1-10\end{array}$ & Composition & Percentage & $\begin{array}{l}\text { Size mm } \\
0.5-3,0\end{array}$ & $\begin{array}{c}\text { Shape } \\
\text { Spherical }\end{array}$ & $\begin{array}{l}\text { Filling } \\
\text { Tron oxide }\end{array}$ & $\begin{array}{c}\text { Percentage } \\
5-15\end{array}$ \\
\hline Plagioclase & $1.0-5.0$ & $\mathrm{An}_{50 \cdot 70}$ & $1-15$ & $\mathrm{An}_{50.65}$ & $25-50$ & & Lobate & Calcite, clay & \\
\hline Clinopyroxene & & & & & $10-35$ & & Irregular & & \\
\hline Mesostasis & & & & & $5-20$ & & & & \\
\hline
\end{tabular}

UNIT 4 Estimate thickness $18.68 \mathrm{~m}$ in Hole $770 \mathrm{C}$ (base at $9 \mathrm{R}-1$, top).

Moderate to Highly Plagioclase-0livine Phyric Pillow Basalt Lavas and Breccias. Hypocrystalline, cryptocrystalline, hyalopilitic and intersertal.

PHENOCRYSTS

\begin{tabular}{|c|c|c|c|c|c|}
\hline FHETUCKSTS & Size $\mathrm{mm}$ & Composition & Percentage & Composition & Percentage \\
\hline Olivine & $0.5-2.0$ & & $1-5$ & & \\
\hline $\begin{array}{l}\text { Plagioclase } \\
\text { Clinopyroxene }\end{array}$ & $1.0-3.0$ & $\mathrm{An}_{70}$ & $5-15$ & $\mathrm{An}_{75}$ & $5-10$ \\
\hline Mesostasis & & & & & 75 \\
\hline
\end{tabular}

VESICLES/AMYGDALES Size $\mathrm{mm} \quad$ Shape Spherical Filling Percentage Plagioclase Mesostasis

UNIT 5 Estimated thickness $10.54 \mathrm{~m}$ in Hole $770 \mathrm{C}$ (base at $10 \mathrm{R}-1,84 \mathrm{~cm}$ ). Moderately Plagioclase-0livine Phyric Basalt Lavas and Dikes. Hypocrystalline, fine-grained, intersertal and intergranular.

PHENOCRYSTS

Olivine

Plagioclase Clinopyroxene Mesostasis

$$
\begin{aligned}
& \begin{array}{lccc}
\text { Size mm } \\
1.0-2.0
\end{array} \quad \text { Composition Percentage Composition Percentage } \begin{array}{c}
\text { Size mm } \\
1.0-3.0
\end{array} \quad \begin{array}{c}
\text { Shape } \\
\text { Spherica }
\end{array} \\
& 2-5
\end{aligned}
$$

UNIT 6 Estimated thickness $11.10 \mathrm{~m}$ in Hole $770 \mathrm{C}$ (base at $11 \mathrm{R}-2,74 \mathrm{~cm}$ ). Sparcely Plagioclase Phyric to Aphyric Dolerite Sill.

Hypocrystalline to holocrystalline, fine-grained, intersertal, intergranular and sub-ophitic

PHENOCRYSTS

GROUNDMASS Percentage $\begin{gathered}\text { VESICLES/AMYGDALES } \\ \text { Size mm Shape }\end{gathered}$
$30-60$
$38-42$

Olivine Size mm Compositio

Percentage Composition

Plagioclase $1.0-2.0$

$$
1-3
$$

$\mathrm{An}_{60}$

UNIT 7 Estimated thickness $10.68 \mathrm{~m}$ in Hole $770 \mathrm{C}$ (base at $12 \mathrm{R}-3,22 \mathrm{~cm}$ ). Sparcely to Highly Plagioclase-olivine Phyric Dolerite Sill. Holocrystalline, fine-grained, intergranular.

PHENOCRYSTS

olivine

Plagioclase c1 inopyroxene Mesostasis

$$
\text { GROUNDMASS }
$$$$
\text { Size } \mathrm{mm} \text { Composition }
$$$$
0.5-3.0
$$$$
\mathrm{An}_{75}
$$$$
\text { Percentage }
$$$$
\begin{aligned}
& 1-15 \\
& 0-5
\end{aligned}
$$$$
\mathrm{An}_{70}
$$

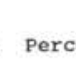

UNIT 8 Estimated thickness is greater than $6.38 \mathrm{~m}$ in Hole $770 \mathrm{C}$ (base not reached). Moderately to Highly Plagioclase-0livine Phyric Basalt Lavas ard Dikes. Hypocrystalline, fine-grained, intersertal and intergranular.

$\begin{array}{lcccc}\text { PHENOCRYSTS } & \text { Size mm } & \text { Composition } & \begin{array}{c}\text { Percentage } \\ \text { GROUNDMASS } \\ \text { Compositio }\end{array} \\ \text { Olivine } & 0.2-10 & & 12 & \\ \text { Plagioclase } & 1.0-4.0 & \mathrm{An}_{80} & 12 & \end{array}$

Plagioclase Mesostasis

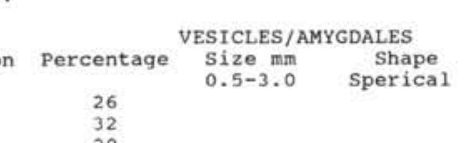

32
20

$$
\begin{aligned}
& \text { VESICLES/AMYGDALES } \\
& \text { SIze mm Shape } \\
& 0.5-3.0 \quad \text { Sperica }
\end{aligned}
$$

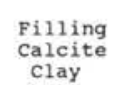

Percentage ratios to classify the basement rocks. They show a very limited range of major-element composition (Table 2 and Shipboard Scientific Party, 1990; Hole 767C Table 18; Holes 770B, 770C Table 13) and strong similarities to average N-MORB composition (from Condie, 1985, Table 1). The $\mathrm{SiO}_{2}$ contents range from $47 \%$ to $50 \%$, thus all of the samples are basalts $\left(<52 \% \mathrm{SiO}_{2}\right.$; LeMaitre, 1976) chemically as well as petrographically, and their $\mathrm{Nb} / \mathrm{Y}$ ratios $(<0.25$, Table 3$)$ show that they are all sub-alkaline (Floyd and Winchester, 1978).
Using the classification of Yoder and Tilley (1962) the majority of the samples in Units 2, 3, 4, 7, and 8 are olivine-normative tholeiites, although a few quartz-tholeiites also occur. In Units 1,5 , and 6 all of the samples are quartz-normative tholeiites (Table 2 and Shipboard Scientific Party, 1990, Hole 767C Table 18 , Hole $770 \mathrm{~B}, 770 \mathrm{C}$ Table 15 ).

Two suites, each with its own distinct chemical characteristics, can be recognized geochemically (Tables 2, 3, 4, and Shipboard Scientific Party, 1990, Hole 767C Table 18, Holes 
Table 2. The average major-element chemistry and normative mineralogy of the basaltic rock units from Holes $767 \mathrm{C}, 770 \mathrm{~B}$, and $770 \mathrm{C}$, Celebes Sea.

\begin{tabular}{|c|c|c|c|c|c|}
\hline $\begin{array}{c}\text { Hole } 770 \\
\text { Core, section } \\
\text { Interval }(\mathrm{cm}) \\
\quad \text { Suite } \\
\text { Unit sample \# }\end{array}$ & $\stackrel{2}{1 . A v}$ & $\begin{array}{c}1 \\
2 . \mathrm{Av}\end{array}$ & $\begin{array}{c}1 \\
\text { 3.Av }\end{array}$ & $\begin{array}{c}770 \mathrm{C} \\
7 \mathrm{R}-5 \\
3-6 \\
1 \\
4.1\end{array}$ & $\begin{array}{c}770 \mathrm{C} \\
7 \mathrm{R}-6 \\
44-47 \\
\text { Int. } \\
5.1\end{array}$ \\
\hline \multicolumn{6}{|c|}{ MAJOR ELEMENT COMPOSITION WT \% } \\
\hline $\begin{array}{l}\mathrm{SiO}_{2} \\
\mathrm{TiO}_{2} \\
\mathrm{Al}_{2} \mathrm{O}_{3} \\
\mathrm{Fe}_{2} \mathrm{O}_{3} \\
\mathrm{MnO} \\
\mathrm{MgO} \\
\mathrm{CaO} \\
\mathrm{Na}_{2} \mathrm{O} \\
\mathrm{K}_{2} \mathrm{O} \\
\mathrm{P}_{2} \mathrm{O}_{5} \\
\mathrm{LOI}\end{array}$ & $\begin{array}{r}48.49 \\
2.26 \\
16.09 \\
10.63 \\
0.23 \\
5.32 \\
11.64 \\
2.71 \\
0.23 \\
0.25 \\
1.84\end{array}$ & $\begin{array}{r}49.11 \\
1.24 \\
18.35 \\
8.05 \\
0.15 \\
6.44 \\
12.55 \\
2.53 \\
0.19 \\
0.10 \\
2.79\end{array}$ & $\begin{array}{r}48.30 \\
1.40 \\
17.33 \\
8.76 \\
0.15 \\
6.84 \\
12.93 \\
2.25 \\
0.33 \\
0.11 \\
4.59\end{array}$ & $\begin{array}{r}49.06 \\
1.29 \\
17.47 \\
9.22 \\
0.15 \\
7.41 \\
12.41 \\
2.33 \\
0.36 \\
0.10 \\
2.77\end{array}$ & $\begin{array}{r}48.32 \\
2.26 \\
16.49 \\
9.75 \\
0.18 \\
5.26 \\
11.48 \\
2.86 \\
0.20 \\
0.24 \\
0.31\end{array}$ \\
\hline $\mathrm{Mg} \#$ & 60.54 & 70.61 & 69.79 & 69.53 & 63.49 \\
\hline $\begin{array}{l}\mathrm{Al}_{2} \mathrm{O}_{3} / \mathrm{TiO}_{2} \\
\mathrm{CaO} / \mathrm{TiO}_{2}\end{array}$ & $\begin{array}{l}7.12 \\
5.15\end{array}$ & $\begin{array}{l}14.80 \\
10.12\end{array}$ & $\begin{array}{r}12.38 \\
9.24\end{array}$ & $\begin{array}{r}13.54 \\
9.62\end{array}$ & $\begin{array}{l}7.30 \\
5.08\end{array}$ \\
\hline \multicolumn{6}{|c|}{ NORMATIVE MINERAL COMPOSITION \% } \\
\hline $\begin{array}{l}\text { Q } \\
\text { Or } \\
\text { Ab } \\
\text { An } \\
\text { Di } \\
\text { Hy } \\
\text { Ol } \\
\text { Mt } \\
\text { Il } \\
\text { Ap }\end{array}$ & $\begin{array}{r}2.75 \\
1.44 \\
25.37 \\
32.38 \\
20.89 \\
9.24 \\
0.00 \\
4.10 \\
3.28 \\
0.56\end{array}$ & $\begin{array}{r}0.39 \\
1.11 \\
23.11 \\
38.82 \\
19.05 \\
12.62 \\
0.00 \\
2.92 \\
1.76 \\
0.22\end{array}$ & $\begin{array}{r}0.00 \\
2.01 \\
20.64 \\
37.11 \\
22.26 \\
12.30 \\
0.35 \\
3.10 \\
1.99 \\
0.24\end{array}$ & $\begin{array}{r}0.00 \\
2.14 \\
21.08 \\
36.46 \\
19.96 \\
13.17 \\
2.23 \\
2.94 \\
1.81 \\
0.21\end{array}$ & $\begin{array}{r}2.70 \\
1.24 \\
26.88 \\
33.07 \\
19.93 \\
8.24 \\
0.00 \\
4.12 \\
3.30 \\
0.53\end{array}$ \\
\hline $\begin{array}{c}\text { Hole } 770 \\
\text { Core, section } \\
\text { Interval }(\mathrm{cm}) \\
\text { Suite } \\
\text { Unit sample \# }\end{array}$ & $\stackrel{2}{2}$ & $\begin{array}{c}770 \mathrm{C} \\
12 \mathrm{R}-2 \\
34-41 \\
1 \\
7.1\end{array}$ & $\begin{array}{c}770 \mathrm{C} \\
12 \mathrm{R}-3 \\
64-66 \\
1 \\
8.1\end{array}$ & $\begin{array}{c}767 \mathrm{C} \\
12 \mathrm{R}-\mathrm{CC} \\
26-28 \\
1\end{array}$ & $\begin{array}{c}767 \mathrm{C} \\
12 \mathrm{R}-\mathrm{CC} \\
36-39 \\
1\end{array}$ \\
\hline \multicolumn{6}{|c|}{ MAJOR ELEMENT COMPOSITION WT \% } \\
\hline $\begin{array}{l}\mathrm{SiO}_{2} \\
\mathrm{TiO}_{2} \\
\mathrm{Al}_{2} \mathrm{O}_{3} \\
\mathrm{Fe}_{2} \mathrm{O}_{3} \\
\mathrm{MnO} \\
\mathrm{MgO} \\
\mathrm{CaO} \\
\mathrm{Na}_{2} \mathrm{O} \\
\mathrm{K}_{2} \mathrm{O} \\
\mathrm{P}_{2} \mathrm{O}_{5} \\
\mathrm{LOI}\end{array}$ & $\begin{array}{r}49.73 \\
2.09 \\
16.09 \\
8.59 \\
0.18 \\
6.27 \\
11.71 \\
2.71 \\
0.21 \\
0.21 \\
1.24\end{array}$ & $\begin{array}{r}47.74 \\
1.33 \\
15.71 \\
10.61 \\
0.22 \\
8.74 \\
11.87 \\
1.79 \\
0.23 \\
0.11 \\
3.22\end{array}$ & $\begin{array}{r}49.06 \\
1.29 \\
17.47 \\
9.22 \\
0.15 \\
7.41 \\
12.41 \\
2.33 \\
0.36 \\
0.10 \\
2.70\end{array}$ & $\begin{array}{r}48.03 \\
1.59 \\
16.87 \\
11.98 \\
0.29 \\
4.99 \\
12.07 \\
2.51 \\
0.29 \\
0.12 \\
1.55\end{array}$ & $\begin{array}{r}49.08 \\
1.59 \\
16.76 \\
9.90 \\
0.31 \\
5.35 \\
12.84 \\
2.28 \\
0.16 \\
0.28 \\
2.31\end{array}$ \\
\hline $\mathrm{Mg} \#$ & 71.29 & 68.99 & 69.53 & 50.94 & 57.40 \\
\hline $\begin{array}{l}\mathrm{Al}_{2} \mathrm{O}_{3} / \mathrm{TiO}_{2} \\
\mathrm{CaO} / \mathrm{TiO}_{2}\end{array}$ & $\begin{array}{l}7.70 \\
5.60\end{array}$ & $\begin{array}{r}11.81 \\
8.92\end{array}$ & $\begin{array}{r}13.54 \\
9.62\end{array}$ & $\begin{array}{r}10.61 \\
7.59\end{array}$ & $\begin{array}{r}10.55 \\
8.08\end{array}$ \\
\hline \multicolumn{6}{|c|}{ NORMATIVE MINERAL COMPOSITION \% } \\
\hline $\begin{array}{l}\mathrm{Q} \\
\mathrm{Or} \\
\mathrm{Ab} \\
\mathrm{An} \\
\mathrm{Di} \\
\mathrm{Hy} \\
\mathrm{Ol} \\
\mathrm{Mt} \\
\mathrm{Il} \\
\mathrm{Ap}\end{array}$ & \begin{tabular}{r|}
3.71 \\
1.28 \\
25.14 \\
32.16 \\
21.18 \\
9.21 \\
0.00 \\
3.87 \\
3.00 \\
0.45
\end{tabular} & $\begin{array}{r}0.00 \\
1.40 \\
16.51 \\
35.12 \\
19.73 \\
20.72 \\
1.34 \\
3.04 \\
1.90 \\
0.24\end{array}$ & $\begin{array}{r}0.00 \\
2.14 \\
21.08 \\
36.46 \\
19.96 \\
13.17 \\
2.23 \\
2.94 \\
1.81 \\
0.21\end{array}$ & $\begin{array}{r}0.00 \\
1.76 \\
23.55 \\
35.47 \\
21.05 \\
11.66 \\
2.38 \\
1.56 \\
2.32 \\
0.27\end{array}$ & $\begin{array}{r}1.75 \\
0.96 \\
21.31 \\
36.49 \\
22.34 \\
12.94 \\
0.00 \\
1.28 \\
2.31 \\
0.61\end{array}$ \\
\hline
\end{tabular}

770B, 770C Tables 13 and 14). The majority of samples (Units $2,3,4,7,8$, and Hole $767 \mathrm{C}$ ) fall into Suite 1, which is characterized by relatively high $\mathrm{Al}_{2} \mathrm{O}_{3}$ contents $(16.49 \%$ $19.23 \%$ ) and $\mathrm{Mg}$-numbers (62.93-72.06) and low concentrations of incompatible minor elements $\left(\mathrm{TiO}_{2}, 1.18 \%-1.60 \%\right.$, $\mathrm{P}_{2} \mathrm{O}_{5}, 0.07 \%-0.17 \%$ ), and trace-elements $(\mathrm{Nb}, 2-5 \mathrm{ppm} ; \mathrm{Y}$, 26-36 ppm; Zr, 67-109 ppm; La, 2.8-5.1 ppm; Yb, 2.8-3.65 ppm). Most of the remaining samples (Units 1 and 6) fall into Suite 2, which is characterized by lower $\mathrm{Al}_{2} \mathrm{O}_{3}(15.67 \%$ $16.49 \%$ ) contents and $\mathrm{Mg}$-numbers (53.77-67.31), and higher incompatible element contents $\left(\mathrm{TiO}_{2}, 2.07 \%-2.32 \% ; \mathrm{P}_{2} \mathrm{O}_{5}\right.$, $0.20 \%-0.27 \%$; Nb, 6-9 ppm; Y, 41-45 ppm; Zr, 141-171 ppm; La, 7.9-8.1 ppm; Yb, 4.3-4.47 ppm) (Tables 2, 3, and 4, and Shipboard Scientific Party, 1990, Hole 767C Table 18, Holes $770 \mathrm{~B}, 770 \mathrm{C}$ Tables 13 and 14) than those in Suite 1. The ranges of $\mathrm{Al}_{2} \mathrm{O}_{3}$ contents and $\mathrm{Mg}$-numbers of the two suites overlap but those of the incompatible minor- and trace-element contents do not. The geochemistry of sample 5.1 is intermediate between that of Suite 1 and Suite 2. Its elemental concentrations may lie in the range of overlap of Suite 1 and Suite 2 $\left(\mathrm{Al}_{2} \mathrm{O}_{3}, 16.49 \%\right)$ or between their ranges of concentration $(\mathrm{Zr}$, $112 \mathrm{ppm}$; La, $5.3 \mathrm{ppm}$; Yb, $3.59 \mathrm{ppm})$, be typical of Suite 1 (Y, $35 \mathrm{ppm})$ or Suite $2\left(\mathrm{TiO}_{2}, 2.26 \% ; \mathrm{P}_{2} \mathrm{O}_{5}, 0.24 \% ; \mathrm{Nb}, 8 \mathrm{ppm}\right)$ contents (Tables 2,3 , and 4). All of the rocks analyzed are characterized by light rare-earth element (LREE)-depleted normalized patterns (Fig. 3). Both suites show limited ranges of $\mathrm{Zr} / \mathrm{TiO}_{2}, \mathrm{Zr} / \mathrm{Y}$, and primordial mantle normalized $(\mathrm{La} /$ $\mathrm{Sm})_{\mathrm{PM}},(\mathrm{La} / \mathrm{Yb})_{\mathrm{PM}}$ ratios and have multielement patterns that lack any significant elemental anomalies (Tables 3 and 4, Figs. 3 and 4). The geochemical signatures of both suites of samples are compared in Table 5 to the characteristic signatures of the mean compositions of ocean-ridge and -floor tholeiites (OFT) (equivalent to N-MORB), oceanic and continental back-arc tholeiites (BAT), tholeiites from island-arcs and continental margins (LKT), enriched mid-ocean ridge basalts (E-MORB), and ocean island tholeiites (OIT) (all from Holm 1985, Tables 2 and 3), and transitional mid-ocean ridge basalt (T-MORB) (from Wood et al., 1979a).

The average composition of Suite 1 is similar in elemental concentration and degree of fractionation $\left((\mathrm{La} / \mathrm{Yb})_{\mathrm{PM}}, 0.66\right)$ to average OFT $\left((\mathrm{La} / \mathrm{Yb})_{\mathrm{PM}}, 0.67\right)$ (Table 5). Suite 2 has higher average concentrations of incompatible elements and a slightly less fractionated REE pattern $\left((\mathrm{La} / \mathrm{Yb})_{\mathrm{PM}}, 0.91\right)$ than those of average OFT (Table 5). The compositional ranges and REE patterns of both suites lie within that of Group 1 (depleted) MORB's (Bryan et al., et al, 1976, Table 3). Both suites have relatively high average $\mathrm{Zr} / \mathrm{Nb}$ ratios (Suite 1, 28.00; Suite 2, 20.68 ), and low average $\mathrm{Zr} / \mathrm{Y}$ ratios (Suite 1, 3.86; Suite 2, 3.55) and slightly LREE-depleted patterns $\left((\mathrm{La} / \mathrm{Yb})_{\mathrm{PM}}\right.$ ratios above). These ratios for both suites (Table 5) lie within or close to the ranges typically associated with normal "depleted" MORB (Le Roex et al, 1983, p. 300, LREE depleted $(\mathrm{La} / \mathrm{Yb})_{\mathrm{N}}, 0.35-1.1$; $\mathrm{Zr} / \mathrm{Nb}, 17-64 ; \mathrm{Zr} / \mathrm{Y}, 1.8-3.6)$.

The following differences are noted in comparison with the other characteristic suites. The average $\mathrm{Nb}$ to $\mathrm{P}$ contents of Suite 1 are similar to those of LKT, but the average $\mathrm{Zr}$ to $\mathrm{Yb}$ contents of Suite 1 are much higher than those of LKT, as are all average elemental contents of Suite 2 (Table 5). The LREE contents, $(\mathrm{La} / \mathrm{Sm})_{\mathrm{PM}}(0.88)$, and $(\mathrm{La} / \mathrm{Yb})_{\mathrm{PM}}(1.33)$ ratios of average BAT are much higher than those of Suite 1 (Table 5), and Suite 2 has much higher concentrations of all incompatible elements than average BAT (Table 5). Neither Suite 1 nor Suite 2 shows the negative $\mathrm{Nb}$ anomalies on primordial mantle normalized multielement plots (Fig. 4), which are typical of arc-related basalts. T-MORB ( $\mathrm{Zr} / \mathrm{Nb}, 9.19), \mathrm{E}-\mathrm{MORB}(\mathrm{Zr} / \mathrm{Nb}$, $6.88)$ and $\mathrm{OIT}(\mathrm{Zr} / \mathrm{Nb}, 8.70)$ have much higher concentrations of $\mathrm{Nb}$ relative to $\mathrm{Zr}$ than both Suite 1 and Suite 2, and 
Table 3. The trace-element contents and ratios of the basaltic rocks from Holes 767C, 770B, and 770C, Celebes Sea.

\begin{tabular}{|c|c|c|c|c|c|}
\hline $\begin{array}{c}\text { Hole } 770 \\
\text { Core-Ssection } \\
\text { Interval }(\mathrm{cm}) \\
\text { Suite } \\
\text { Unit Sample \# }\end{array}$ & $\begin{array}{c}\mathrm{B} \\
16 \mathrm{R}-4 \\
84-87 \\
2 \\
1.1\end{array}$ & $\begin{array}{c}\text { B } \\
17 \mathrm{R}-3 \\
134-137 \\
2 \\
1.2\end{array}$ & $\begin{array}{c}\mathrm{C} \\
2 \mathrm{R}-3 \\
59-62 \\
2 \\
1.4\end{array}$ & $\begin{array}{c}\mathrm{C} \\
3 \mathrm{R}-2 \\
125-128 \\
2 \\
1.5\end{array}$ & $\stackrel{2}{1 . \mathrm{Av}}$ \\
\hline \multicolumn{6}{|c|}{ TRACE ELEMENT CONTENTS (ppm) } \\
\hline $\begin{array}{l}\mathrm{V} \\
\mathrm{Cr} \\
\mathrm{Co} \\
\mathrm{Ni} \\
\mathrm{Cu} \\
\mathrm{Zn} \\
\mathrm{Rb} \\
\mathrm{Sr} \\
\mathrm{Y} \\
\mathrm{Zr} \\
\mathrm{Nb} \\
\mathrm{Ba}\end{array}$ & $\begin{array}{r}118 \\
63 \\
98 \\
3 \\
3 \\
195 \\
44 \\
165 \\
16 \\
6 \\
5\end{array}$ & $\begin{array}{r}115 \\
64 \\
100 \\
2 \\
186 \\
45 \\
171 \\
7 \\
3\end{array}$ & $\begin{array}{r}335 \\
222 \\
75 \\
95 \\
62 \\
104 \\
5 \\
187 \\
44 \\
165 \\
9 \\
7\end{array}$ & $\begin{array}{r}348 \\
234 \\
62 \\
117 \\
60 \\
106 \\
5 \\
193 \\
45 \\
168 \\
7 \\
62\end{array}$ & $\begin{array}{r}339 \\
223 \\
69 \\
111 \\
62 \\
102 \\
4 \\
190 \\
45 \\
167 \\
7 \\
19\end{array}$ \\
\hline \multicolumn{6}{|c|}{ TRACE ELEMENT RATIOS } \\
\hline $\begin{array}{l}\mathrm{Zr} / \mathrm{TiO}_{2} \\
\mathrm{Zr} / \mathrm{Y} \\
\mathrm{Zr} / \mathrm{Nb} \\
\mathrm{Y} / \mathrm{Nb} \\
\mathrm{Mg} \#\end{array}$ & $\begin{array}{c}0.0074 \\
3.75 \\
27.50 \\
7.33 \\
54.97\end{array}$ & $\begin{array}{c}0.0074 \\
3.80 \\
24.43 \\
6.43 \\
53.77 \\
\end{array}$ & $\begin{array}{c}0.0074 \\
3.75 \\
18.33 \\
4.89 \\
57.03 \\
\end{array}$ & $\begin{array}{c}0.0074 \\
3.73 \\
24.00 \\
6.43 \\
57.38 \\
\end{array}$ & $\begin{array}{c}0.0074 \\
3.76 \\
23.07 \\
6.43\end{array}$ \\
\hline $\begin{array}{c}\mathrm{B} \\
19 \mathrm{R}-1 \\
99-102 \\
1 \\
2.2 \\
\end{array}$ & $\begin{array}{c}\mathrm{B} \\
19 \mathrm{R}-2 \\
42-45 \\
1 \\
2.3 \\
\end{array}$ & $\begin{array}{c}\text { B } \\
20 \mathrm{R}-2 \\
102-105 \\
1 \\
2.4 \\
\end{array}$ & $\begin{array}{c}\mathrm{B} \\
20 \mathrm{R}-3 \\
10-15 \\
1 \\
2.5 \\
\end{array}$ & $\begin{array}{c}\mathrm{C} \\
4 \mathrm{R}-1 \\
27-30 \\
1 \\
2.6\end{array}$ & $\begin{array}{c}1 \\
2 . \mathrm{Av}\end{array}$ \\
\hline $\begin{array}{r}150 \\
76 \\
87 \\
3 \\
111 \\
31 \\
70 \\
3 \\
24\end{array}$ & $\begin{array}{r}144 \\
78 \\
76 \\
3 \\
107 \\
29 \\
67 \\
67 \\
2 \\
5\end{array}$ & $\begin{array}{r}154 \\
80 \\
69 \\
1 \\
111 \\
26 \\
69 \\
2 \\
24\end{array}$ & $\begin{array}{r}286 \\
302 \\
205 \\
74 \\
72 \\
1 \\
108 \\
28 \\
69 \\
2 \\
9\end{array}$ & $\begin{array}{r}233 \\
329 \\
54 \\
132 \\
68 \\
75 \\
3 \\
124 \\
31 \\
84 \\
4 \\
6\end{array}$ & $\begin{array}{r}270 \\
351 \\
54 \\
157 \\
75 \\
76 \\
2 \\
112 \\
29 \\
72 \\
3 \\
14\end{array}$ \\
\hline $\begin{array}{l}0.0055 \\
2.26 \\
23.33 \\
10.33 \\
63.86 \\
\end{array}$ & $\begin{array}{l}0.0057 \\
2.31 \\
33.50 \\
14.50 \\
62.93 \\
\end{array}$ & $\begin{array}{l}0.0056 \\
2.65 \\
34.50 \\
13.00 \\
71.87 \\
\end{array}$ & $\begin{array}{l}0.0057 \\
2.46 \\
34.50 \\
14.00 \\
72.06 \\
\end{array}$ & $\begin{array}{c}0.0068 \\
2.71 \\
21.00 \\
7.75 \\
64.45 \\
\end{array}$ & $\begin{array}{l}0.0058 \\
2.48 \\
27.62 \\
11.92\end{array}$ \\
\hline $\begin{array}{c}\text { B } \\
20 \mathrm{R}-4 \\
135-140 \\
1 \\
3.1\end{array}$ & $\begin{array}{c}\text { B } \\
21 \mathrm{R}-1 \\
96-99 \\
1 \\
3.2\end{array}$ & $\begin{array}{c}\text { B } \\
21 \mathrm{R}-4 \\
7-77 \\
1 \\
3.4\end{array}$ & $\begin{array}{c}\text { B } \\
21 \mathrm{R}-6 \\
15-18 \\
1 \\
3.5\end{array}$ & $\begin{array}{c}\mathrm{C} \\
5 \mathrm{R}-2 \\
49-51 \\
1 \\
3.6\end{array}$ & $\begin{array}{c}\mathrm{C} \\
6 \mathrm{R}-3 \\
45-48 \\
1 \\
3.7\end{array}$ \\
\hline $\begin{array}{l}237 \\
352\end{array}$ & $\begin{array}{l}263 \\
244\end{array}$ & $\begin{array}{l}260 \\
263\end{array}$ & $\begin{array}{l}244 \\
238\end{array}$ & $\begin{array}{r}227 \\
316 \\
60\end{array}$ & $\begin{array}{r}260 \\
301 \\
59\end{array}$ \\
\hline $\begin{array}{r}104 \\
93 \\
84 \\
2\end{array}$ & $\begin{array}{r}249 \\
73 \\
78 \\
9\end{array}$ & $\begin{array}{r}190 \\
80 \\
81 \\
6\end{array}$ & $\begin{array}{r}152 \\
76 \\
78 \\
6\end{array}$ & $\begin{array}{r}136 \\
81 \\
75 \\
10\end{array}$ & $\begin{array}{r}116 \\
74 \\
87 \\
11\end{array}$ \\
\hline $\begin{array}{r}114 \\
29\end{array}$ & $\begin{array}{r}129 \\
31\end{array}$ & $\begin{array}{r}138 \\
33\end{array}$ & $\begin{array}{r}141 \\
31\end{array}$ & $\begin{array}{r}111 \\
29\end{array}$ & $\begin{array}{r}139 \\
34\end{array}$ \\
\hline 72 & 86 & 97 & 95 & 79 & 100 \\
\hline $\begin{array}{l}3 \\
1\end{array}$ & $\begin{array}{r}3 \\
28\end{array}$ & $\begin{array}{r}3 \\
16\end{array}$ & $\begin{array}{r}3 \\
13\end{array}$ & $\begin{array}{r}5 \\
36\end{array}$ & $\begin{array}{l}5 \\
4\end{array}$ \\
\hline $\begin{array}{l}0.0059 \\
2.48 \\
24.00 \\
9.67\end{array}$ & $\begin{array}{l}0.0062 \\
2.77 \\
28.67 \\
10.33\end{array}$ & $\begin{array}{l}0.0061 \\
2.94 \\
32.33 \\
11.00\end{array}$ & $\begin{array}{l}0.0064 \\
3.06 \\
31.67 \\
10.33\end{array}$ & $\begin{array}{l}0.0063 \\
2.72 \\
15.80 \\
5.80\end{array}$ & $\begin{array}{c}0.0065 \\
2.94 \\
20.00 \\
6.80\end{array}$ \\
\hline 67.44 & 67.92 & 65.22 & 66.16 & 66.52 & 65.30 \\
\hline
\end{tabular}

Table 3 (continued).

\begin{tabular}{|c|c|c|c|c|c|}
\hline $\begin{array}{c}1 \\
\text { 3.Av }\end{array}$ & $\begin{array}{c}\mathrm{C} \\
7 \mathrm{R}-5 \\
3-6 \\
1 \\
4.1\end{array}$ & $\begin{array}{c}\mathrm{C} \\
7 \mathrm{R}-6 \\
44-47 \\
\text { Int. } \\
5.1\end{array}$ & $\begin{array}{c}\mathrm{C} \\
10 \mathrm{R}-2 \\
43-47 \\
2 \\
6.1\end{array}$ & $\begin{array}{l}\mathrm{C} \\
11 \mathrm{R}-1 \\
91-93 \\
2 \\
6.2\end{array}$ & $\stackrel{2}{2}$ 6. \\
\hline 213 & 280 & 290 & 317 & 335 & 326 \\
\hline 245 & 320 & 298 & 230 & 235 & 233 \\
\hline 60 & 74 & 73 & 66 & 70 & 68 \\
\hline 135 & 136 & 95 & 109 & 110 & 110 \\
\hline 68 & 39 & 54 & 67 & 65 & 66 \\
\hline 69 & 94 & 93 & 104 & 94 & 99 \\
\hline 6 & 10 & 13 & 6 & 4 & 5 \\
\hline 110 & 132 & 141 & 158 & 158 & 158 \\
\hline 27 & 34 & 35 & 43 & 41 & 42 \\
\hline 76 & 109 & 112 & 152 & 141 & 147 \\
\hline 3 & 5 & 8 & 8 & 8 & 8 \\
\hline 14 & 8 & 1 & 49 & 45 & 47 \\
\hline 0.0054 & 0.0084 & 0.0070 & 0.0073 & 0.0067 & 0.0074 \\
\hline 2.83 & 3.21 & 3.20 & 3.53 & 3.44 & 3.49 \\
\hline 24.05 & 21.80 & 14.00 & 19.00 & 17.63 & 18.31 \\
\hline \multirow[t]{2}{*}{9.00} & 6.80 & 4.38 & 5.38 & 5.13 & 5.25 \\
\hline & 66.73 & 57.38 & 61.82 & 67.31 & \\
\hline $\mathrm{C}$ & $\mathrm{C}$ & $767 \mathrm{C}$ & $767 C$ & & \\
\hline $12 \mathrm{R}-2$ & $12 \mathrm{R}-3$ & 12R-CC & $12 \mathrm{R}-\mathrm{CC}$ & & \\
\hline $34-41$ & $64-66$ & $26-28$ & $36-39$ & & \\
\hline 1 & 1 & 1 & 1 & & \\
\hline 7.1 & 8.1 & & & & \\
\hline 270 & 227 & 386 & 344 & & \\
\hline 387 & 322 & 344 & 353 & & \\
\hline 64 & 68 & & & & \\
\hline 173 & 162 & 172 & 171 & & \\
\hline 53 & 75 & 71 & 28 & & \\
\hline 69 & 76 & 107 & 201 & & \\
\hline 7 & 9 & 5 & 3 & & \\
\hline 105 & 108 & 124 & 123 & & \\
\hline 32 & 29 & 33 & 36 & & \\
\hline 90 & 80 & 91 & 92 & & \\
\hline 3 & 3 & 2 & 2 & & \\
\hline 43 & 55 & 12 & 13 & & \\
\hline 0.0068 & 0.0062 & 0.0057 & 0.0057 & & \\
\hline 2.81 & 2.76 & 2.76 & 2.56 & & \\
\hline 30.00 & 26.67 & 45.50 & 46.00 & & \\
\hline 10.67 & 9.67 & 16.50 & 18.00 & & \\
\hline 67.27 & 66.73 & 50.94 & 57.40 & & \\
\hline
\end{tabular}

Note: Samples with Co values were determined at Laurentian University.

E-MORB $\left((\mathrm{La} / \mathrm{Yb})_{\mathrm{PM}}, 2.92\right)$ and OIT $\left((\mathrm{La} / \mathrm{Yb})_{\mathrm{PM}}, 4.15\right)$ have LREE-enriched patterns rather than the depleted patterns of both Suite 1 and Suite 2 (Table 5).

The Celebes Sea may be a trapped fragment of the Indian Ocean floor (Lee and McCabe, 1986). N-type (depleted) MORB, T-type (transitional) MORB, and P-type (plume or enriched) MORB suites have all been reported from the Indian Ocean, commonly in close association. The range of LREEdepleted immobile element compositions reported for Indian Ocean N-MORB's (Bryan et al., 1976, Fig. 5; Frey et al., 1980, Fig. 4; Subbarao and Reddy, 1981, Fig. 2; Le Roex et al., 1983, Figs. 2, 3, and 6; Price et al., 1986) is sufficiently large to include the total range of composition of both Suite 1 and Suite 2. Suite 1 is comparable to the N-MORB described by Price et al., 1986, Tables 4 and 5) from within $70 \mathrm{~km}$ of the Indian Ocean triple junction (IORa, Table 5). Suite 2 basalts are more difficult to classify. A detailed comparison shows that Sample 36-7 (IORb, Table 5) which is classified as an N-MORB (Le Roex et al., 1983, Table 2, pp. 271-272), is very similar in composition to the average composition of Suite 2, but has slightly more fractioned REE patterns $\left((\mathrm{La} / \mathrm{Sm})_{\mathrm{PM}}\right.$ 
A

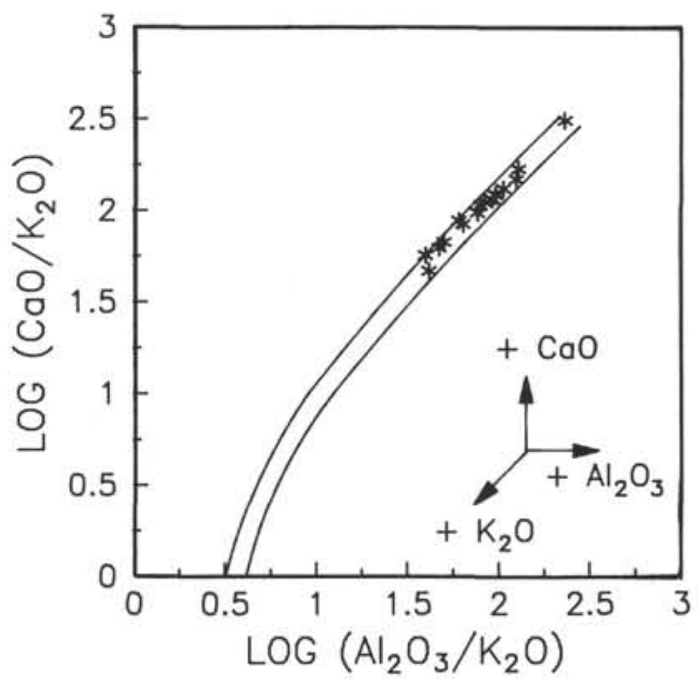

B

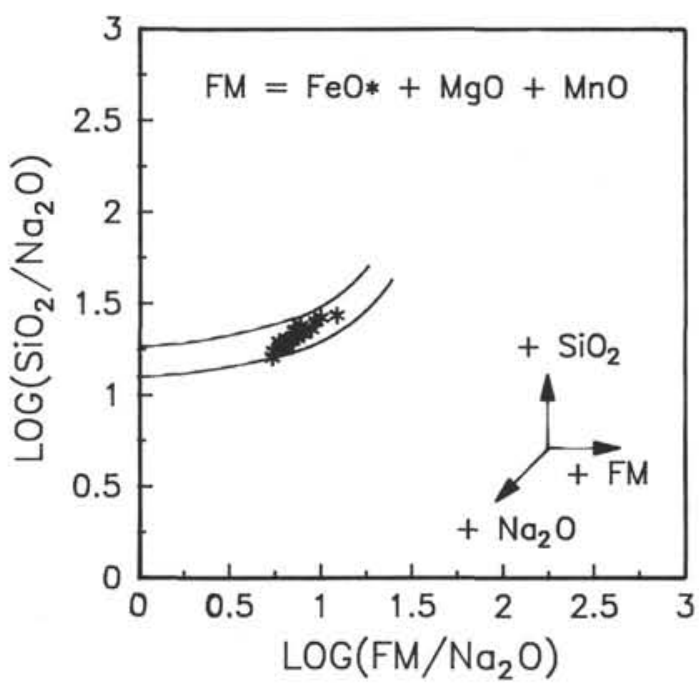

Figure 2. Selected Beswick and Soucie (1978) diagrams illustrating the chemical changes due to alteraton of the rocks from Sites 767 and 770 , Celebes Sea. The boundary curves enclose the fields of unaltered rocks and the arrows represent vectors of secondary enrichment. A. The $\log \left(\mathrm{CaO} / \mathrm{K}_{2} \mathrm{O}\right)$ vs. $\log \left(\mathrm{Al}_{2} \mathrm{O}_{3} / \mathrm{K}_{2} \mathrm{O}\right)$ illustrates the increase in $\mathrm{K}_{2} \mathrm{O}$ contents. B. The $\log \left(\mathrm{SiO}_{2} / \mathrm{Na}_{2} \mathrm{O}\right)$ vs. $\log \left(\mathrm{FM} / \mathrm{Na}_{2} \mathrm{O}\right)$ illustrates the increase in $\mathrm{Na}_{2} \mathrm{O}$ contents.

$\left.1.01,(\mathrm{La} / \mathrm{Yb})_{\mathrm{PM}} 1.28\right)$. In addition, they have higher $\mathrm{Zr} / \mathrm{Nb}$ and $\mathrm{Y} / \mathrm{Nb}$ ratios (14-23, and 4.4-6, respectively) and lower (La/ $\mathrm{Yb})_{\mathrm{PM}}$ ratios $(0.79-0.98)$, as compared to typical ranges reported for T-MORB's from the Indian Ocean (Le Roex et al., $1983, \mathrm{Zr} / \mathrm{Nb}$ 7.7-11.8, Y/Nb 1.3-3.0, $\left.(\mathrm{La} / \mathrm{Yb})_{\mathrm{N}} 1.7-4.3\right)$. Price et al., (1986) report a suite of T-MORB's (IORc, Table 5) that occurs at distances of greater than $250 \mathrm{~km}$ from the Indian Ocean triple junction, which have lower concentrations of $\mathrm{Nb}$, $\mathrm{La}, \mathrm{Sm}, \mathrm{Ti}, \mathrm{Y}$, and $\mathrm{Yb}$, but more fractionated REE patterns $\left((\mathrm{La} / \mathrm{Yb})_{\mathrm{PM}} 1.27\right)$, than those of Suite 2. On balance, the rocks of Suite 2 are considered to be N-MORB's. Thus all of the rocks recovered from Holes $767 \mathrm{C}, 770 \mathrm{~B}$, and $770 \mathrm{C}$ are identified as N-type (depleted) MORB's.
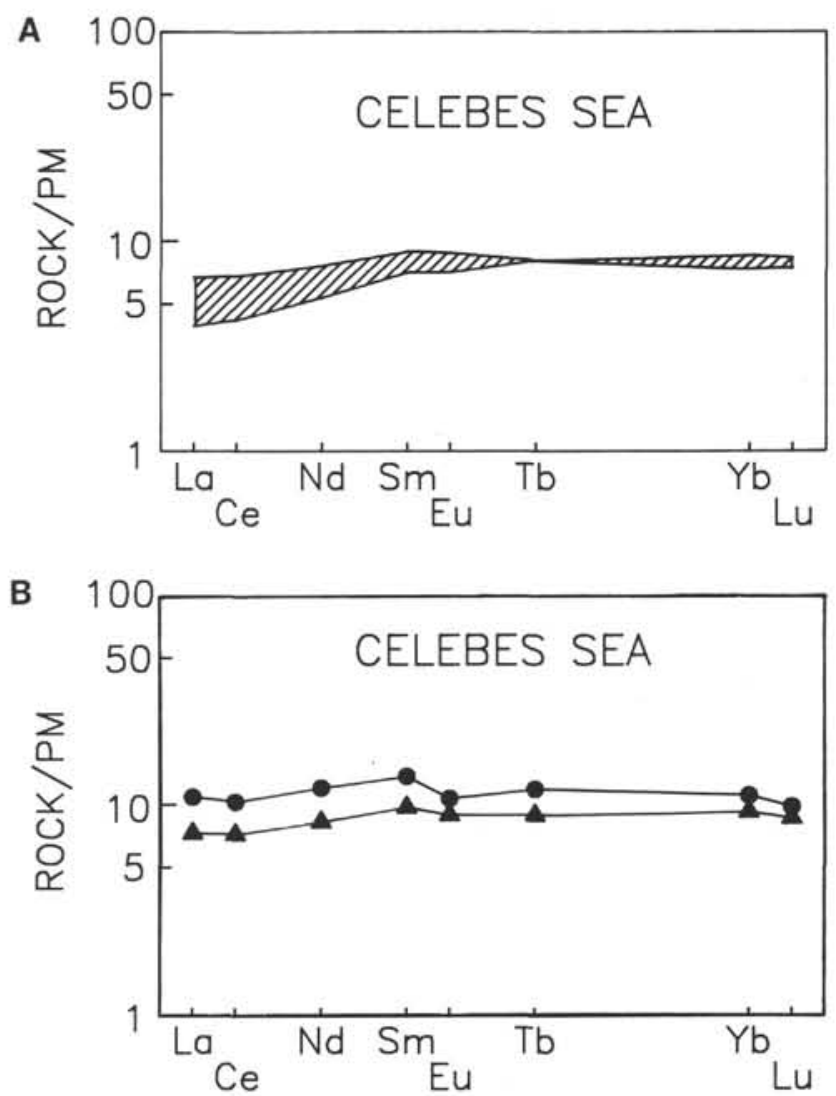

Figure 3. Primordial mantle-normalized (Wood et al., 1979a) rareearth element plot of basaltic rocks from Sites 767 and 770 . A. Shows the range of variation in Suite 1, lower limits defined by sample 2.3, upper limits by sample 3.5. B. Suite 2 sample 1.1 (solid circle), and intermediate sample 5.1 (solid triangle).

\section{DISCUSSION}

The purpose of this section is to show that all of the Celebes Sea basement rocks can be formed from one LREEdepleted source by processes similar to those generally considered to yield MORB's. Multielement plots of rock suites reflect the mineralogical and chemical composition of their source materials, as well as their partial melting, fractional crystallization, and mixing histories (Thompson et al., 1983). There are differences in the concentrations of the incompatible elements between Suite 1 and Suite 2 but their multielement distribution patterns are very similar (Fig. 4). This similarity suggests that they are genetically related (Thompson et al., 1986), and the differences in incompatible element concentrations may result from different partial melting histories and/or fractional crystallization histories. The effects of both of these processes can be identified in the basalts recovered from the Celebes Sea.

Suite 1 has systematically lower $\mathrm{TiO}_{2}$ contents and higher $\mathrm{Al}_{2} \mathrm{O}_{3} / \mathrm{TiO}_{2}$ and $\mathrm{CaO} / \mathrm{TiO}_{2}$ ratios and $\mathrm{Mg}$-numbers than Suite 2 (Table 2). This inverse relationship, taken together with the similarities in their trace-element ratios, suggests that both rock suites were formed by different degrees of partial melting of the same, or similar, source materials (Sun et al., 1979 and Subbarao and Veddy, 1981). Higher degrees of partial melting progressively eliminate the major aluminiumand calcium-bearing phases from the mantle source, thus increasing the contents of $\mathrm{Al}_{2} \mathrm{O}_{3}$ and $\mathrm{CaO}$ and their ratios to $\mathrm{TiO}_{2}$ in the melts. The concentrations of $\mathrm{TiO}_{2}$ and other 
A

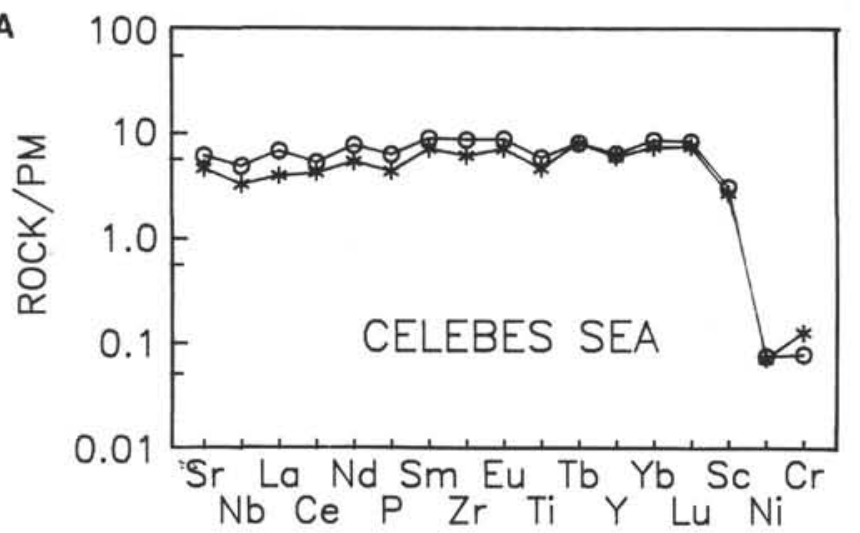

B

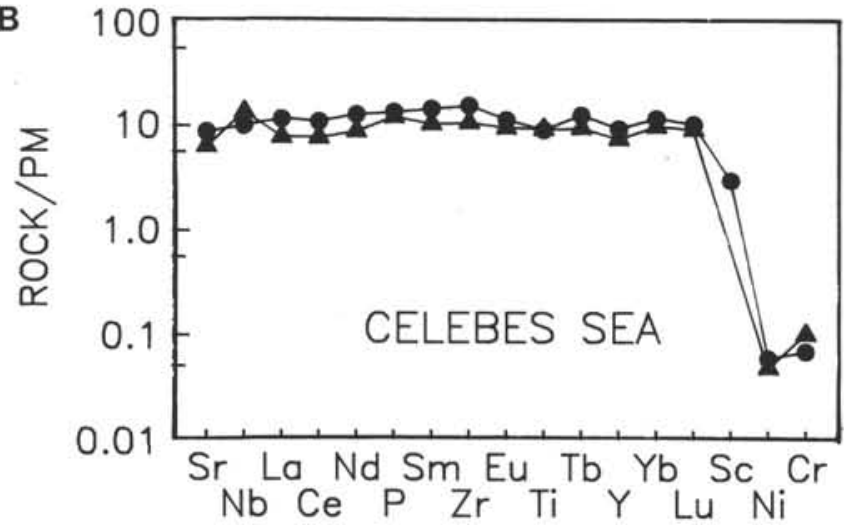

Figure 4. Primordial mantle-normalized (Wood et al., 1979a) multielement plot of the basaltic rocks from Holes 767C, 770B, and 770C, Celebes Sea. A. Suite 1 unevolved sample 2.3 (asterisk), evolved sample 767C (open circle), B. Suite 2 sample 1.1 (solid circle), intermediate sample 5.1 (solid triangle).

incompatible elements are reduced in proportion to the degree of melting, thereby preserving the incompatible trace-element ratios in the resultant rocks (Suites 1 and 2). The presence of phenocrysts of olivine and plagioclase, in the basalts of both suites (Table 1), is evidence that fractional crystallization has affected the melts from which the rocks were formed. The rocks of Suite 1 are characterized by relatively high $\mathrm{Mg}$-number (60 to 70 ) and low contents of $\mathrm{TiO}_{2}(1.25 \%), \mathrm{P}_{2} \mathrm{O}_{5}(0.10 \%)$, and incompatible trace elements. Suite 2 is characterized by lower $\mathrm{Mg}$ numbers ( 53 to $60)$ and high concentrations of $\mathrm{TiO}_{2}(2.25 \%), \mathrm{P}_{2} \mathrm{O}_{5}(0.25 \%)$, and incompatible elements (Tables 2, 3, and 4, and Shipboard Scientific Party, 1990; Holes 770B, 770C Tables 13 and 14). Such large differences in incompatible element contents, with such small differences in $\mathrm{Mg}$ numbers, cannot be produced by fractional crystallization. Within each suite there are small decreases in Mg-numbers coupled with small increases in incompatible trace-element contents and small decreases in compatible element contents (Tables 2, 3, and 4), which suggest that each suite has undergone a limited amount of evolution through fractional crystallization.

The complex stratigraphic relationships of Suites 1 and 2 are helpful in choosing the type of melting model. The two suites are interdigitated at various leveis in the stratigraphic sequence (Table 1), and each suite occurs as pillow lavas, massive flows, and sills. Each suite is also characterized by different $\mathrm{Mg}$ numbers and different concentrations of trace elements. The multielement patterns of the two suites do not cross. These relationships indicate that the rock suite cannot
Table 4. The rare-earth element contents and ratios of selected basaltic rocks from Sites 767 and 770, Celebes Sea. $(\mathrm{La} / \mathrm{Sm})_{\mathrm{PM}}$ and $(\mathrm{La} / \mathrm{Yb})_{\mathrm{PM}}$ are primordial mantle normalized ratios.

\begin{tabular}{|c|c|c|c|c|}
\hline $\begin{array}{c}\text { Hole } 770 \\
\text { Core-Section } \\
\text { Interval }(\mathrm{cm}) \\
\text { Suite } \\
\text { Unit Sample \# }\end{array}$ & $\begin{array}{c}\text { B } \\
16 \mathrm{R}-4 \\
84-87 \\
2 \\
1.1\end{array}$ & $\begin{array}{c}\mathrm{B} \\
19 \mathrm{R}-2 \\
42-45 \\
1 \\
2.3\end{array}$ & $\begin{array}{c}\text { B } \\
20 \mathrm{R}-2 \\
102-105 \\
1 \\
2.4\end{array}$ & $\begin{array}{c}\text { B } \\
20 R-4 \\
105-135 \\
1 \\
3.1\end{array}$ \\
\hline \multicolumn{5}{|c|}{ TRACE ELEMENTS (ppm) } \\
\hline $\begin{array}{l}\mathrm{Sc} \\
\mathrm{La} \\
\mathrm{Ce} \\
\mathrm{Nd} \\
\mathrm{Sm} \\
\mathrm{Eu} \\
\mathrm{Tb} \\
\mathrm{Yb} \\
\mathrm{Lu} \\
\mathrm{Th} \\
\mathrm{U}\end{array}$ & $\begin{array}{l}38 \\
7.9 \\
20 \\
16 \\
5.38 \\
1.62 \\
1.2 \\
4.3 \\
0.59 \\
0.3 \\
0.7\end{array}$ & $\begin{array}{l}35 \\
2.8 \\
8 \\
7 \\
2.73 \\
1.05 \\
0.8 \\
2.8 \\
0.44 \\
0.6 \\
0.1\end{array}$ & $\begin{array}{l}2.9 \\
8 \\
7 \\
2.73 \\
1.13 \\
0.7 \\
2.89 \\
0.41 \\
0.2 \\
0.1\end{array}$ & $\begin{array}{l}38 \\
3.3 \\
9 \\
7 \\
2.81 \\
1.03 \\
0.7 \\
2.88 \\
0.42 \\
0.3 \\
0.5\end{array}$ \\
\hline \multicolumn{5}{|c|}{ ELEMENTAL RATIOS } \\
\hline $\begin{array}{l}(\mathrm{La} / \mathrm{Sm})_{\mathrm{PM}} \\
(\mathrm{La} / \mathrm{Yb})_{\mathrm{PM}} \\
\end{array}$ & $\begin{array}{l}0.80 \\
0.98\end{array}$ & $\begin{array}{l}0.56 \\
0.54\end{array}$ & $\begin{array}{l}0.58 \\
0.54\end{array}$ & $\begin{array}{l}0.64 \\
0.61\end{array}$ \\
\hline $\begin{array}{c}\text { B } \\
21 \mathrm{R}-1 \\
96-99 \\
1 \\
3.2\end{array}$ & $\begin{array}{c}\mathrm{B} \\
21 \mathrm{R}-6 \\
15-18 \\
1 \\
3.5\end{array}$ & $\begin{array}{c}\mathrm{C} \\
7 \mathrm{R}-6 \\
44-47 \\
\text { Int } \\
5.1\end{array}$ & $\begin{array}{c}\mathrm{C} \\
10 \mathrm{R}-2 \\
43-47 \\
2 \\
6.1\end{array}$ & $\begin{array}{c}767 \mathrm{C} \\
12 \mathrm{R}-\mathrm{CC} \\
26-28 \\
1\end{array}$ \\
\hline $\begin{array}{l}39 \\
4.1 \\
11 \\
9 \\
3.13 \\
1.18 \\
0.8 \\
3.05 \\
0.44 \\
0.2 \\
0.3\end{array}$ & $\begin{array}{l}40 \\
4.8 \\
10 \\
10 \\
3.44 \\
1.3 \\
0.8 \\
3.26 \\
0.49 \\
0.2 \\
0.2\end{array}$ & $\begin{array}{l}5.3 \\
14 \\
11 \\
3.82 \\
1.35 \\
0.9 \\
3.59 \\
0.52 \\
0.2 \\
0.2\end{array}$ & $\begin{array}{l}8.1 \\
18 \\
15 \\
5.01 \\
1.85 \\
1.2 \\
4.47 \\
0.66 \\
0.4 \\
0.1\end{array}$ & $\begin{array}{l}48 \\
5.1 \\
11 \\
10 \\
3.8 \\
1.39 \\
1 \\
3.65 \\
0.54 \\
0.2 \\
0.2\end{array}$ \\
\hline $\begin{array}{l}0.71 \\
0.72\end{array}$ & $\begin{array}{l}0.76 \\
0.79\end{array}$ & $\begin{array}{l}0.75 \\
0.79\end{array}$ & $\begin{array}{l}0.88 \\
0.97\end{array}$ & $\begin{array}{l}0.73 \\
0.75\end{array}$ \\
\hline
\end{tabular}

be explained in terms of frequent replenishement and tapping of a magma chamber, as suggested by O'Hara (1977) to explain certain MORB suites, or by the selective dynamic melting model.

Tholeiitic magmas are generally considered to form by $15 \%$ to $30 \%$ partial melting of the mantle, although a few workers consider that all mantle-derived magmas form by very small degrees of partial melting (Thompson et al., 1984). Primordial mantle and chondrite compositions were tested as possible trace-element source compositions. Within the generally accepted limits of partial melting, the major, compatible $(\mathrm{Ni}, \mathrm{Cr}$, $\mathrm{Sc})$, and incompatible trace-element contents $(\mathrm{Zr}, \mathrm{Nb}, \mathrm{Y}, \mathrm{Ti}$, and REE) can best be obtained by partial melting of a source with a primordial mantle trace-element composition (Wood et al., 1979a). Partial melting of sources having chondritic traceelement contents can only yield reasonable matches of the trace-element chemistry of the suites at less than $10 \%$ partial melting.

The modal mineralogy and the melting proportions of the source were based on the generalized models for the formation of MORB's (Condie, 1985, and Pearce, 1985) modified according to the $\mathrm{Ni}, \mathrm{Cr}$, and $\mathrm{Sc}$ contents of the resultant melts (Leeman, 1976). The major element contents and the distribution of $\mathrm{Ni}$ and $\mathrm{Cr}$ in the Celebes rocks (Hanson and Langmuir, 1978) suggest that Suite 1 and Suite 2 were formed 
Table 5. A comparison of the average elemental contents and ratios of selected basaltic rocks from Suite 1 and Suite 2 Sites 767 and 770 , Celebes Sea, with those of rock suites erupted in various tectonic environments. OFT, mean ocean floor tholeiites; BAT, mean back-arc tholeiites; LKT, mean low-potassium tholeiites; E-MORB, mean enriched mid-ocean ridge basalts; OIT, mean ocean island tholeiites, from Holm, 1985, Tables 2 and 3; T-MORB, transitional mid-ocean ridge basalt from Wood et al., 1979b; IORa, average N-MORB Indian Ocean triple junction, Price et al., 1986, Tables 4 and 5; IORb, N-MORB from Bouvet Fracture Zone, Le Roex et al., 1983, Table 2 (sample 36-7); IORc, average T-MORB $250 \mathrm{~km}$ from Indian Ocean triple junction, Price et al., Tables 4 and 5; IORd, T-MORB Islas Orcadas, Southwest Indian Ridge, Le Roex et al., 1983, Table 7 (average of samples 62-30 and 62-49). $(\mathrm{La} / \mathrm{Sm})_{\mathrm{PM}}$ and $(\mathrm{La} / \mathrm{Y})_{\mathrm{PM}}$ are primordial mantle-normalized ratios.

\begin{tabular}{lcccccc}
\hline & SUITE 1 & SUITE 2 & OFT & BAT & LKT & T-MORB \\
\hline TRACE ELEMENTS $(\mathrm{ppm})$ & & & & & \\
$\mathrm{Nb}$ & 2 & 7.4 & 4.35 & 3.22 & 1.26 & 12.3 \\
$\mathrm{La}$ & 2.8 & 7.1 & 3.6 & 5.68 & 2.9 & 6.1 \\
$\mathrm{Ce}$ & 8 & 17.3 & 10.5 & 15 & 8.22 & 19 \\
$\mathrm{P}$ & 437 & 1037 & 615 & 669 & 470 & 960 \\
$\mathrm{Zr}$ & 112 & 153 & 96 & 105 & 37 & 113 \\
$\mathrm{Sm}$ & 2.73 & 4.7 & 3.19 & 3.5 & 1.65 & 4.71 \\
$\mathrm{Ti}$ & 7434 & 13286 & 8704 & 7635 & 4581 & 10431 \\
$\mathrm{Y}$ & 29 & 43 & 35 & 26.8 & 15.6 & 38.4 \\
$\mathrm{Yb}$ & 2.8 & 4.12 & 3.28 & 2.58 & 1.58 & 3.94 \\
& & & & & & \\
ELEMENTAL & $\mathrm{RATIOS}$ & & & & & \\
$(\mathrm{La} / \mathrm{Sm})_{\text {PM }}$ & 0.66 & 0.81 & 0.61 & 0.88 & 0.95 & 0.70 \\
$(\mathrm{La} / \mathrm{Yb}$ ) & 0.66 & 0.91 & 0.67 & 1.33 & 1.11 & 0.83 \\
\hline
\end{tabular}

\begin{tabular}{lcccccc}
\hline & E-MORB & OIT & IORa & IORb & IORc & IORd \\
\hline \multicolumn{1}{c}{ TRACE } & ELEMENTS $(\mathrm{ppm})$ & & & & & \\
$\mathrm{Nb}$ & 20.5 & 20 & 2.2 & 9.2 & 4.3 & 16.9 \\
$\mathrm{La}$ & 13.5 & 15.8 & 3.08 & 9.9 & 5.6 & 14 \\
$\mathrm{Ce}$ & 34 & 38 & 10.9 & 20.7 & 17.3 & 29.8 \\
$\mathrm{P}$ & 1175 & 1284 & 666 & 1048 & 1149 & 1069 \\
$\mathrm{Zr}$ & 141 & 174 & 93 & 154 & 139 & 145 \\
$\mathrm{Sm}$ & 5.4 & 6.24 & 3.04 & 4.98 & 3.52 & 4.67 \\
$\mathrm{Ti}$ & 12063 & 15728 & 7764 & 12230 & 6475 & 12050 \\
$\mathrm{Y}$ & 28.7 & 30.7 & 30.1 & 42 & 29 & 33 \\
$\mathrm{Yb}$ & 2.8 & 2.27 & 3.02 & 4.34 & 2.67 & 3.22 \\
& & & & & & \\
$\mathrm{ELEMENTAL}$ & $\mathrm{RATIOS}$ & & & & & \\
$(\mathrm{La} / \mathrm{Sm})$ & 1.36 & 1.36 & 0.55 & 1.01 & 0.87 & 1.64 \\
$(\mathrm{La} / \mathrm{Yb}$ ) & 2.92 & 4.15 & 0.55 & 1.28 & 1.27 & 2.63 \\
\hline
\end{tabular}

respectively by approximately $20 \%$ and $10 \%$ batch partial melting of a peridotitic mantle followed by olivine fractionation. The flat HREE patterns point to partial melting of a spinel lherzolite source, and relatively low $\mathrm{Ni}$ and $\mathrm{Cr}$ contents are most readily produced when a small amount of spinel remains after the melt has been generated. Both rock suites contain phenocrysts of olivine and plagioclase.

The normalized ratios of the LREE's are lower than those of the heavy rare-earth elements (HREE) in all of the samples analyzed (Suite 1, $(\mathrm{La} / \mathrm{Sm})_{\mathrm{PM}} 0.66,(\mathrm{La} / \mathrm{Yb})_{\mathrm{PM}} 0.66$, Suite 2, $\left.(\mathrm{La} / \mathrm{Sm})_{\mathrm{PM}} 0.81,(\mathrm{La} / \mathrm{Yb})_{\mathrm{PM}} 0.91\right)$ and point toward the derivation of the melts from a LREE-depleted source. The HFSE, HREE, and incompatible element contents of the rocks were used to determine the percentages of partial melting and fractionation involved in their formation. The LREE contents of the primordial mantle source were then modified to produce the best overall fit for both suites. This approach produced a more realistic petrogenetic model than would be the case if source compositions were tailored to each suite individually (Thompson et al., 1986, p. 5989). Three samples, 2.3, 2.4, and Hole $767 \mathrm{C}$, were chosen as representative of Suite 1 for modeling. The composition of the least-evolved rock in Suite 1 (2.3) can be produced by approximately $20 \%$ partial melting
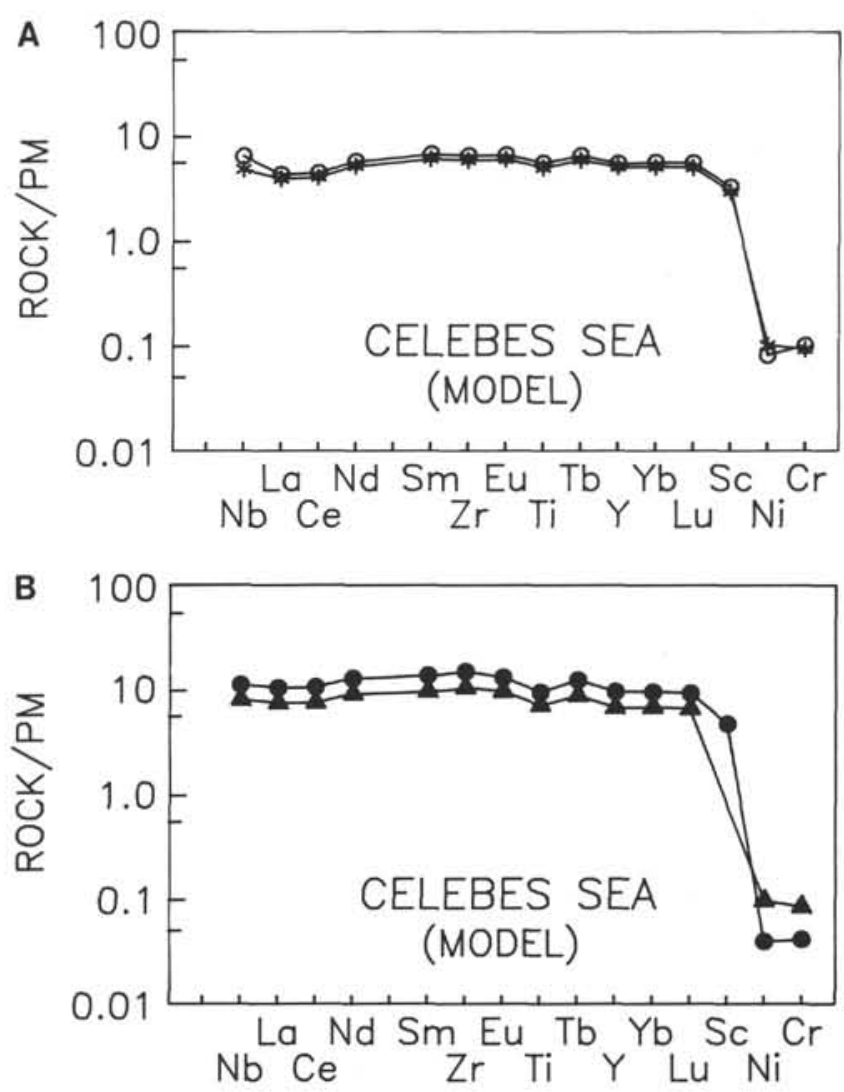

Figure 5. Primordial mantle-normalized plots (Wood et al., 1979) of the modeled compositions of selected samples compared with the measured values plotted in Figure 4. A. Suite 1 sample 2.3 (asterisk), and $767 \mathrm{C}$ (open circle), B. Suite 2 sample 1.1 (solid circle), and intermediate sample 5.1 (solid triangle) between the two suites.

of primordial mantle (Wood et al., 1979a) in which the LREE have been depleted, followed by $20 \%$ olivine fractionation. Sample 2.4, from Suite 1, may be produced by an additional $10 \%$ fractional crystallization of a 60:40 mixture of olivine and plagioclase. The most-evolved sample from Suite 1 was collected from Hole 767C and may be produced from sample 2.4 by an additional $30 \%$ of fractional crystallization of the $60: 40$ olivine:plagioclase mixture (Fig. 5A).

There is a limited range of variation in the trace-element composition in Suite 2 and sample 1.1 is chosen as representative for modeling. This sample has a relatively low $\mathrm{Mg}$ number and may be modeled in terms of $10 \%$ partial melting of the LREE-depleted primordial mantle source, followed by two stages of fractional crystallization, $20 \%$ removal of olivine, and $30 \%$ removal of a mixture of olivine $(58 \%)$, plagioclase (40\%), and magnetite (2\%) (Fig. 5B).

Details of the model used are as follows.

All modeled melts are formed from the same spinel lherzolite source by different degrees of partial melting and fractional crystallization. Source mode ol $60 \%$, opx $26 \%$, cpx $12 \%$, sp $2 \%$. Source elemental composition is LREE-depleted primordial mantle (Wood et al., 1979b). Depletions are La $63 \%$, Ce $66 \%$, and Nd $85 \%$ of Wood's values. Suite 1 is formed by $20 \%$ partial melting (residue ol $73 \%$, opx $25 \%$, cpx $0 \%$, sp $3 \%$ ) followed by $20 \%$ fractional crystallization of ol to yield 2.3. The composition of 2.4 , which is not illustrated, can be formed from 2.3 by $10 \%$ more fractional crystallization of the olivine plagioclase mixture. A further $30 \%$ fractional crystallization of ol $60 \%$ plus pl $40 \%$ yields $767 \mathrm{C}$. Suite 2 is 
Table 6. Distribution coefficients used in the modeling calculations after Arth (1976), Irving (1978), Pearce and Norry (1979), and Condie (pers. comm.).

\begin{tabular}{lccrc}
\hline Mafic & Ol & Opx & Cpx & PI \\
\hline $\mathrm{La}$ & 0.00001 & 0.007 & 0.070 & 0.150 \\
$\mathrm{Ce}$ & 0.00001 & 0.008 & 0.100 & 0.120 \\
$\mathrm{Nd}$ & 0.00007 & 0.010 & 0.220 & 0.081 \\
$\mathrm{Sm}$ & 0.00060 & 0.020 & 0.400 & 0.067 \\
$\mathrm{Eu}$ & 0.00100 & 0.020 & 0.400 & 0.350 \\
$\mathrm{~Tb}$ & 0.00200 & 0.050 & 0.500 & 0.060 \\
$\mathrm{Yb}$ & 0.02000 & 0.150 & 0.600 & 0.070 \\
$\mathrm{Lu}$ & 0.01600 & 0.180 & 0.600 & 0.060 \\
$\mathrm{Y}$ & 0.01000 & 0.010 & 0.200 & 0.030 \\
$\mathrm{Zr}$ & 0.01000 & 0.030 & 0.030 & 0.010 \\
$\mathrm{Nb}$ & 0.01000 & 0.010 & 0.020 & 0.010 \\
$\mathrm{Ti}$ & 0.02000 & 0.100 & 0.180 & 0.040 \\
$\mathrm{Ni}$ & 5.00000 & 3.100 & 2.500 & 0.000 \\
$\mathrm{Cr}$ & 0.10000 & 5.000 & 15.000 & 0.000 \\
$\mathrm{Sc}$ & 0.16000 & 0.160 & 0.510 & 0.040 \\
\hline
\end{tabular}

\begin{tabular}{rrrr}
\hline Gnt & \multicolumn{1}{c}{ Spl } & \multicolumn{1}{l}{ Hbd } & Mgt \\
\hline 0.030 & 0.000 & 0.200 & 0.000 \\
0.030 & 0.000 & 0.260 & 0.000 \\
0.087 & 0.000 & 0.400 & 0.000 \\
0.220 & 0.000 & 0.700 & 0.000 \\
1.000 & 0.001 & 0.800 & 0.000 \\
3.000 & 0.002 & 0.800 & 0.000 \\
5.000 & 0.020 & 0.600 & 0.000 \\
5.500 & 0.016 & 0.500 & 0.000 \\
2.000 & 0.010 & 1.000 & 0.200 \\
0.300 & 0.010 & 1.500 & 0.100 \\
0.100 & 0.010 & 0.800 & 0.400 \\
0.300 & 0.800 & 1.500 & 7.500 \\
5.100 & 16.000 & 7.000 & 30.000 \\
17.500 & 600.000 & 15.000 & 150.000 \\
2.270 & 2.000 & 1.500 & 2.000 \\
\hline
\end{tabular}

formed by $10 \%$ partial melting (residue ol $66 \%$, opx $26 \%$, cpx $7 \%$, sp $2 \%$ ) followed by $20 \%$ fractional crystallization of ol, and $30 \%$ fractional crystallization of $58 \% \mathrm{ol}, 40 \% \mathrm{pl}$, and $2 \%$ $\mathrm{mt}$ yields 1.1 . Intermediate rock 5.1 is formed by $14 \%$ partial melting, followed by $20 \%$ fractional crystallization of ol and a further $40 \%$ fractional crystallization of a mixture $59 \%$ ol, $40 \%$ $\mathrm{pl}$ and $1 \% \mathrm{mt}$. Distribution coefficients used in the modeling are given in Table 6; Abbreviations: ol olivine, opx orthopyroxene, cpx clinopyroxene, sp spinel, pl plagioclase, $\mathrm{mt}$ magnetite (magnetite is used in the calculations in the absence of partition coefficients for Fe-Ti oxide).

Sample 5.1 is intermediate in its trace-element characteristics between Suite 1 and Suite 2. Its composition may be matched by $14 \%$ partial melting of the same LREE-depleted primordial mantle, followed by $20 \%$ removal of olivine and a further $40 \%$ removal of a $60: 40$ mixture of olivine and plagioclase (Fig. 5B).

Thus the basalts making up the basement of the Celebes Sea are similar to N-MORB's in composition. The variations in their chemistry can be accounted for in terms of different degrees of partial melting of a spinel lherzolite source having a LREEdepleted primordial mantle trace-element composition and different percentages of fractional crystallization of olivine/plagioclase mixtures. These processes are typical of those generally believed to be involved in the evolution of MORB's.

\section{MORB-CELEBES COMPARISON}

The MORB-like charcteristics of these samples support the contention that the Celebes Sea was once continuous with the late Jurassic Argo abyssal plain, as suggested by Lee and McCabe (1986) on the basis of magnetic anomalies, heat-flow rates, and bathymetry. In the same study Lee and McCabe (1986) suggested that the Banda Basin to the south, and the Sulu Sea to the north, of the Celebes Sea were also relics of a Cretaceous-Eocene oceanic crust. They suggested that it was possible to identify magnetic anomalies 30 to 33 in the Celebes Sea, indicating that the basin was formed in the Paleocene (65-72 m.y. BP). However, the basaltic basement of the Celebes Sea is directly overlain by radiolarian-bearing red pelagic clay containing fish teeth and manganese micronodules of middle Eocene to Upper Oligocene age. This confirms that the magnetic anomalies present are numbers 18-20 (42-47 m.y. BP Weissel, 1980) and that the basin was formed during Eocene times. Rangin (1989) has suggested that the Sulu and Celebes basins have different origins. He used onshore and offshore data to show that the Celebes plate subducted northward beneath a continental margin, along which an Oligocene-to-early Miocene arc developed. This arc rifted to form the Sulu Sea basin by back-arc spreading, leaving the Cagayan Ridge as a remnant (inactive) arc. Drilling in the Sulu Sea basin has produced data that confirm that it is a back-arc basin formed in late early Miocene times, and that continental arc volcanism ceased on the the Cagayan Ridge more or less simultaneously with the formation of the basin, and it became a remnant arc (Smith et al., this volume). The identification of middle Eocene N-MORB basalts in the Celebes Sea, and of middle Micocene back-arc tholeiites in the Sulu Sea strongly support Rangin's (1989) interpretation.

\section{CONCLUSIONS}

The basement of the Celebes Sea comprises a series of middle Eocene basaltic rocks having N-MORB-like compositions, very similar to rocks formed at the Indian Ocean triple junction. This fact suggests that the basement represents a trapped fragment of a once larger ocean basin. Regional considerations suggest that the Celebes Plate subducted to the north beneath the edge of the Eurasian continental plate, and that this motion led to the formation of the Sulu Sea by back-arc rifting during the middle Miocene.

\section{ACKNOWLEDGMENTS}

We thank the Natural Sciences and Engineering Research Council of Canada for providing funds for this study, and Drs. M. Fisk, R. Taylor, and T. Alabaster for their helpful reviews of the manuscript.

\section{REFERENCES}

Abbott, P. L., and Smith, T. E., 1978. Trace element comparison of clasts in Eocene conglomerates, southwestern California and northwestern Mexico. J. Geol.,86:753-762.

Arth, J. G., 1976. Behaviour of trace elements during magmatic processes: a summary of theoretical models and their applications. J. Res. U.S. Geol. Surv.,4:41-47.

Augustithis, S. S., 1979. Atlas of the Textural Patterns of Basic and Ultrabasic Rocks and Their Genetic Significance: New York (Walter de Gruyter).

Basaltic Volcanism Study Project. 1981. Basaltic Volcanism on the Terrestrial Planets: New York (Pergamon Press).

Beswick, A. E., and Soucie, G., 1978. A correction procedure for metasomatism in Archean greenstone belts. Precambrian Res., 6:235-248.

Bryan, W. B., Thompson, G., Frey, F. A., and Dickey, J. S., 1976. Inferred geologic settings and differentiation in basalts from the deep-sea drilling project. J. Geophys. Res.,81:4285-4304.

Condie, K. C., 1977. Archean Greenstone Belts: Developments in Precambrian Geology Ser Vol. 3: New York (Elsevier).

1985. Secular variation in the composition of basalts: an index to mantle evolution. J. Petrol.,26:545-563.

Condie, K. C., Bowling, G. P., and Vance, R. K., 1985. Geochemistry and origin of the early Proterozoic rocks, Dos Cabezas Mountains, southeastern Arizona. Geol. Soc. Am. Bull.,96:655-662. 
Floyd, P. A., and Winchester, J. A., 1978. Identification and discrimination of altered and metamorphosed volcanic rocks using immobile elements. Chem. Geol., 21:291-306.

Frey, F. A., Dickey, J. S., Jr., Thompson, G., Bryan, W. B., and Davis, H. L., 1980. Evidence for heterogeneous primary MORB and mantle sources, northwest Indian Ocean. Contrib. Miner. Petrol.,74:387-402.

Gelinas, L., Mellinger, M., and Trudel, P., 1982. Archean mafic metavolcanics from the Rouyn-Noranda district, Abitibi Greenstone Belt, Quebec. 1. Mobility of the major elements. Can. J. Earth Sci.,19:2258-2275.

Hanson, G. N., and Langmuir, C. H., 1978. Modelling of major elements in mantle-melt systems using trace element approaches. Geochim. Cosmochim. Acta,42:725-741.

Holm, P. E., 1985. The geochemical fingerprints of different tectonomagmatic environments using hygromagmatophile element abundances of tholeiitic basalts and basaltic andesites. Chem. Geol.,51:303-323.

1988. Petrogenetic modeling with a spreadsheet program. J. Geol. Educ.,36:155-156.

Holm, P. E., in press. Complex petrogenetic modeling using spreadsheet software. Comput. Geosci.

Huang, C. H., and Smith, T. E., 1983. Application of the linear relationship between the reciprocal of the analyte-line intensity and the reciprocal of concentration to the analysis of geological materials. $X$-Ray Spectrom., 12:87-90.

Irving, A. J., 1978. A review of experimental studies of crystal/liquid trace element partitioning. Geochim. Cosmochim. Acta,42:743770 .

Kokelaar, B. P., Howells, M. F., Bevins, R. E., Roach, R. A., and Dunkley, P. N., 1984. The Ordovician marginal basin of Wales. In Kokelaar, B. P., and Howells, M. F. (Eds.), Marginal Basin Geology. Geol. Soc. Spec. Publ. London, 16:245-270.

Lee, C. S., and McCabe, R., 1986. The Banda-Celebes-Sulu basin: a trapped piece of Cretaceous oceanic crust? Nature,322:51-54.

Leeman, W. P., 1976. Petrogenesis of McKinney (Snake River) olivine tholeiite in the light of rare earth and $\mathrm{Cr} / \mathrm{Ni}$ distributions. Geol. Soc. Am. Bull.,87:1582-1586.

Le Maitre, R. W., 1976. The chemical variability of some common igneous rocks. J. Petrol., 17:589-637.

Leg 124 Shipboard Scientific Party, 1989. Origins of marginal basins. Nature, 338:380-381.

Le Roex, A. P., Dick, H.J.B., Erlank, A. J., Reid, A. M., Frey, F. A., and Hart, S. R., 1983. Geochemistry, mineralogy and petrogenesis of lavas erupted along southwest Indian Ridge between the Bouvet Triple Junction and 11 Degrees East. J. Petrol., 24:267-318.

Ludden, J., Gelinas, L., and Trudel, P., 1982. Archean metavolcanics from the Rouyn-Noranda district, Abitibi Greenstone Belt, Quebec. 2 . Mobility of trace elements and petrogenetic constraints. Can. J. Earth Sci.,19:2276-2287.

O'Hara, M. J., 1977. Geochemical evolution during fractional crystallization of a periodically refilled magma chamber. Nature, 266:503-507.

Pearce, J. A., 1983. Role of the sub-continental lithosphere in magma genesis at active continental margins. In Hawkesworth, C. J., and Norey, M. J. (Eds.), Continental basalts and mantle xenoliths: Cheshire, U.K. (Shiva Publishing Ltd.), 230-249.

Pearce, J. A., Lippard, S. J., and Roberts, S., 1984. Characteristics and tectonic significance of supra-subduction zone ophiolites. In Kokelaar, B. P., and Howells, M. F. (Eds.), Marginal Basin Geology. Geol. Soc. Spec. Publ. London, 16:59-76.

Pearce, J. A., and Norry, M. J., 1979. Petrogenetic implications of Ti, $\mathrm{Zr}, \mathrm{Y}$, and $\mathrm{Nb}$ variations in volcanic rocks. Contrib. Mineral. Petrol., 69:33-47.

Peck, D. C., and Smith, T. E., 1989. The geology and geochemistry of an early Proterozoic volcanic-arc association at Cartwright Lake: Lynn Lake Greenstone Belt, Northwestern Manitoba. Can. J. Earth Sci., 26:716-736.
Price, R. C., Kennedy, A. K., Riggs-Sneeringer, M., and Frey, F. A., 1986. Geochemistry of basalts from the Indian Ocean triple junction: implications for the generation and evolution of Indian Ocean ridge basalts. Earth Planet. Sci. Lett., 78:379-396.

Rangin, C., 1989. The Sulu Sea, a back-arc basin setting within a Neogene collision zone. Tectonophysics, 161:119-141.

Rautenschlien, M., Jenner, G. A., Hertogen, J., Hoffmann, A. W., Kerrich, R., Schmincke, H. U., and White, W. M., 1985. Isotopic and trace element composition of volcanic glasses from Akaki Canyon, Cyprus: implications for the origin of the Troodos ophiolite. Earth Planet. Sci. Lett.,75:369-383.

Saunders, A. D., and Tarney, J., 1984. Geochemical characteristics of basaltic volcanism within back-arc basins. In Kokelaar, B. P., and Howells, M. F. (Eds.), Marginal Basin Geology. Geol. Soc. Spec. Publ. London, 16:59-76.

Shipboard Scientific Party, 1990. In Rangin, C., Silver, E. A., von Breymann, M. T., et al., Proc. ODP, Init. Repts., 124: College Station, TX (Ocean Drilling Program).

Subbarao, K. V., and Reddy, V. V., 1981. Geochemical studies on oceanic basalts from the Indian Ocean. Tectonophysics., 75:6989.

Sun, S. S., Nesbitt, R. W., and Sharaskin, A. Ya., 1979. Geochemical characteristics of mid-ocean ridge basalts. Earth Planet. Sci. Lett.,44:119-138.

Thompson, R. N., Morrison, M. A., Dickin, A. P., and Hendry, G. L., 1983. Continental flood basalts ..... . arachnids rule. In Hawkesworth, C. J., and Norry, M. J. (Eds.), Continental Basalts and Mantle Xenoliths: Nantwich, U. K. (Shiva), 158-185.

Thompson, R. N., Morrison, M. A., Hendry, G. L., and Parry, S. J., 1984. An assessment of the relative roles of crust and mantle in magma genesis: an elemental approach. Philos. Trans. R. Soc. London, A310:549-590.

Thompson, R. N., Morrison, M. A., Dickin, A. P., Gibson, I. L., and Harmon, R. S., 1986. Two contrasting styles of interaction between basic magmas and continental crust in the British Tertiary Volcanic Province. J. Geophys. Res., 91:5985-5997.

Wakita, H., Rey, P., and Schmitt, R. A., 1971. Abundances of the 14 rare-earth elements and 12 other trace-elements in Apollo 12 samples: five igneous and one breccia rocks and four soils. Proc. 2nd Lunar Sci. Conf., 1319-1329.

Walker, D., Shibats, T., and Delong, S., 1979. Abyssal tholeiites from the Oceanographer Fracture Zone. Contrib. Mineral. Petrol., $70: 111-125$.

Weissel, J. K., 1980. The tectonic and geological evolution of southeast asian seas and islands. In Hayes, D. E. (Ed.), The Tectonic and Geologic Evolution of Southeast Asian Seas and Islands (Pt. 2): Am. Geophys. Union Monogr., 23:37-47.

Wood, D. A., Joron, J. L., and Treuil, M., 1979a. A re-appraisal of the use of trace elements to classify and discriminate between magma series erupted in different tectonic settings. Earth Planet. Sci. Lett., 45:326-336.

Wood, D. A., Joron, L. L., Treuil, M., Norry, M., and Tarney, J., 1979b. Elemental and $\mathrm{Sr}$ isotope variations in basic lavas from Iceland and the surrounding ocean floor. Contrib. Mineral. Petrol.,70:319-339.

Yoder, H. S., and Tilley, C. E., 1962. Origin of basaltic magmas: an experimental study of natural and synthetic rock systems. $J$. Petrol.,3:342-532.

Date of initial receipt: 1 June 1990

Date of acceptance: 1 November 1990

Ms 124B-161 